\title{
Taylor Expansions of Jacobi Forms and Applications to Explicit Structures of Degree Two
}

\author{
by
}

Tomoyoshi IBUkIYAma

\begin{abstract}
Natural mappings from the coefficients of the Taylor expansion of Jacobi forms of general degree to products of certain spaces of vector valued Siegel modular forms are constructed. Proving surjectivity of these mappings in some special cases, we also clarify explicit structures of Jacobi forms of degree two of index one and of even weight of index two as modules over the graded ring of Siegel modular forms of even weight. We also prove surjectivity of the diagonal restriction of Jacobi forms of index one of degree two to the symmetric tensors of Jacobi forms of degree one.
\end{abstract}

2010 Mathematics Subject Classification: Primary 11F50; Secondary 11F46.

Keywords: Jacobi forms, vector valued Siegel modular forms, differential operators.

\section{$\S 1$. Introduction}

In this paper, we first give a characterization of the coefficients of the Taylor expansion of Jacobi forms of general degree with respect to the second variable in $\mathbb{C}^{n}$ by vector valued Siegel modular forms. This is a generalization of a result of Eichler and Zagier [5] on the relation between the Taylor coefficients of Jacobi forms of degree one and modular forms of degree one. Secondly we apply it to determine explicit structures of Jacobi forms of degree two of index one of any weight and of index two of even weight. A short announcement of the results in this paper has been published in [13].

More precise contents are as follows. We denote by $H_{n}$ the Siegel upper halfspace of degree $n$. Jacobi forms $F(\tau, z)$ of degree $n$ are functions of $(\tau, z) \in H_{n} \times \mathbb{C}^{n}$ which have the same automorphic properties as the functions appearing as coefficients of the Fourier expansion of Siegel modular forms of degree $n+1$ with respect

Communicated by A. Tamagawa. Received August 10, 2011. Revised December 15, 2011.

T. Ibukiyama: Department of Mathematics, Graduate School of Science, Osaka University, Machikenayama 1-1, Toyonaka, Osaka, 560-0043 Japan;

e-mail: ibukiyam@math.sci.osaka-u.ac.jp 
to the $(n+1, n+1)$-component of $H_{n+1}$. A systematic extensive study was done in Eichler-Zagier's book [5] in the case $n=1$. In this paper, we show the following three results.

(1) For general degree $n$, there exist linear maps of the Taylor coefficients of $F(\tau, z)$ at $z=0$ to direct products of vector valued Siegel modular forms of certain weights (cf. Theorem 3.1).

(2) We apply (1) to give explicit structures of the modules of Jacobi forms of $\Gamma_{2}^{J}=S p(2, \mathbb{Z})^{J}$ (the Jacobi modular group of degree two) of index one, and of even weight of index two, over the ring of Siegel modular forms of degree two of even weights (cf. Theorems 5.1, 6.1).

(3) We also show that the Witt operator (the restriction map $H_{2} \times \mathbb{C}^{2} \rightarrow$ $\left.\left(H_{1} \times \mathbb{C}\right)^{2}\right)$ maps surjectively Jacobi forms of degree two of index one onto the symmetric tensors of Jacobi forms of degree one of index one (cf. Theorem 7.1).

Among the assertions in (2), the result for the index one case has been essentially given before in [7] by using correspondence with Siegel modular forms of half-integral weight (see [8]), but here we give a simple alternative direct proof.

In degree one case, it is known from [5] how many Taylor coefficients of a Jacobi form $F$ determine $F$. Indeed, denote by $J_{k, m}\left(\Gamma_{n}^{J}\right)$ the space of Jacobi forms of degree $n$ of weight $k$ of index $m$ with respect to the Jacobi modular group $\Gamma_{n}^{J}:=S p(n, \mathbb{Z})^{J}$ defined in Section 2 , and by $J_{k, m}^{(i)}\left(\Gamma_{n}^{J}\right)$ the subspace of those such that all the Taylor coefficients vanish up to total degree $i$. Then if $n=1$, it is easy to see that $J_{k, m}^{(2 m)}\left(\Gamma_{1}^{J}\right)=0$ (cf. [5]). But this is not true for general $n$. The following problem is open in general.

Problem 1.1. What is the smallest $i$ such that $J_{k, m}^{(i)}\left(\Gamma_{n}^{J}\right)=0$ ?

For example, we have $J_{k, 1}^{(2)}\left(\Gamma_{2}^{J}\right)=0$ by Theorem 4.1 but $J_{k, 2}^{(4)}\left(\Gamma_{2}^{J}\right) \neq 0$ by Theorem 5.1 and we can also show $J_{k, 1}^{(2)}\left(\Gamma_{3}^{J}\right) \neq 0$. It will be interesting to study this problem for more general cases.

\section{$\S 2$. Jacobi forms and Siegel modular forms}

We recall several definitions here. We first define vector valued Siegel modular forms. We denote by $S p(n, \mathbb{R})$ the symplectic group of rank $n$ defined by

$$
S p(n, \mathbb{R})=\left\{g \in M_{2 n}(\mathbb{R}) ; g J_{n}{ }^{t} g=J_{n}\right\},
$$

where $J_{n}=\left(\begin{array}{cc}0_{n} & -1_{n} \\ 1_{n} & 0_{n}\end{array}\right)$ and $1_{n}$ is the unit matrix of size $n$. We denote by $\Gamma_{n}$ the Siegel modular group of level one defined by $\Gamma_{n}=S p(n, \mathbb{R}) \cap M_{2 n}(\mathbb{Z})$. For any finitedimensional rational representation $(\rho, V)$ of $G L_{n}(\mathbb{C})$, any $V$-valued function $F(\tau)$ 
on $H_{n}$, and any element $g=\left(\begin{array}{ll}a & b \\ c & d\end{array}\right) \in S p(n, \mathbb{R})$, we write

$$
\left(\left.F\right|_{\rho}[g]\right)(\tau)=\rho(c \tau+d)^{-1} F(g \tau)
$$

A $V$-valued holomorphic function $F(\tau)$ on $H_{n}$ is called a Siegel modular form of weight $\rho$ with respect to $\Gamma_{n}$ if $\left.F\right|_{\rho}[\gamma]=F$ for all $\gamma \in \Gamma_{n}$; when $n=1, F(t)$ is moreover assumed to be holomorphic at the cusp $i \infty$. We denote by $A_{\rho}\left(\Gamma_{n}\right)$ the vector space of Siegel modular forms defined above. In this article, we mainly treat the case when the weight is $\rho_{k, \nu}=\operatorname{det}^{k} \mathrm{Sym}_{\nu}$, the tensor product of $\operatorname{det}^{k}$ and the symmetric tensor representation $\operatorname{Sym}_{\nu}$ of degree $\nu$. When $\rho=\rho_{k, \nu}$ we write $A_{\rho}\left(\Gamma_{n}\right)=A_{k, \nu}\left(\Gamma_{n}\right)$, and if $\nu=0$, we write $A_{\rho}\left(\Gamma_{n}\right)=A_{k}\left(\Gamma_{n}\right)$, which is the space of usual scalar valued Siegel modular forms of weight $k$. We denote by $S_{k, \nu}\left(\Gamma_{n}\right)$ or $S_{k}\left(\Gamma_{n}\right)$ the subspace of cusp forms. When $F \in A_{k, \nu}\left(\Gamma_{n}\right)$, by the action of $-1_{2 n} \in \Gamma_{n}$, we have $F(\tau)=(-1)^{n k+\nu} F(\tau)$. So $A_{k, \nu}\left(\Gamma_{n}\right)=0$ unless $n k+\nu$ is even.

We fix a realization of $\rho_{k, \nu}$ as follows. We denote by $V_{\nu}$ the vector space of homogeneous polynomials $P(u)$ in $n$ variables $u=\left(u_{1}, \ldots, u_{n}\right)$ of total degree $\nu$, and the representation $\rho_{k, \nu}$ is defined by $\rho_{k, \nu}(g): P(u) \mapsto \operatorname{det}(g)^{k} P(u g)$ for $g \in$ $G L_{n}(\mathbb{C})$. For $\alpha=\left(\alpha_{1}, \ldots, \alpha_{n}\right) \in\left(\mathbb{Z}_{\geq 0}\right)^{n}$ and $u=\left(u_{1}, \ldots, u_{n}\right)$, we write $u^{\alpha}=$ $\prod_{i=1}^{n} u_{i}^{\alpha_{i}}$. We also write $|\alpha|=\sum_{i=1}^{n} \alpha_{i}$. Then a holomorphic $V_{\nu}$-valued function $F$ is identified with

$$
F=\sum_{|\alpha|=\nu} f_{\alpha}(\tau) u^{\alpha}
$$

where $f_{\alpha}(\tau)$ are scalar valued holomorphic functions. To emphasize that it is a polynomial of $u$, we sometimes write $F=F(\tau, u)$. The automorphy of $F \in$ $A_{k, \nu}\left(\Gamma_{n}\right)$ means

$$
F(g \tau, u)=\operatorname{det}(c \tau+d)^{k} F(\tau, u(c \tau+d)) .
$$

Or if we write $u$ as a column vector, this relation can also be written as

$$
F\left(g \tau,{ }^{t}(c \tau+d)^{-1} u\right)=\operatorname{det}(c \tau+d)^{k} F(\tau, u)
$$

Example: When $n=\nu=2, g=\left(\begin{array}{cc}A & B \\ C & D\end{array}\right) \in \Gamma_{2}, C \tau+D=\left(\begin{array}{ll}\alpha & \beta \\ \gamma & \delta\end{array}\right)$, and $F(\tau, u)=$ $f_{20}(\tau) u_{1}^{2}+f_{11}(\tau) u_{1} u_{2}+f_{02}(\tau) u_{2}^{2} \in A_{k, 2}\left(\Gamma_{2}\right)$, we have

$$
\left(\begin{array}{l}
f_{20}(g \tau) \\
f_{11}(g \tau) \\
f_{02}(g \tau)
\end{array}\right)=\operatorname{det}(c \tau+d)^{k}\left(\begin{array}{ccc}
\alpha^{2} & \alpha \beta & \beta^{2} \\
2 \alpha \gamma & \alpha \delta+\beta \gamma & 2 \beta \delta \\
\gamma^{2} & \gamma \delta & \delta^{2}
\end{array}\right)\left(\begin{array}{l}
f_{20}(\tau) \\
f_{11}(\tau) \\
f_{02}(\tau)
\end{array}\right)
$$


Next we recall the definition of Jacobi forms. We define the Jacobi modular group of degree $n$ as the subgroup of $\Gamma_{n+1}$ given by

$$
\Gamma_{n}^{J}=\left\{\left(\begin{array}{llll}
a & 0 & b & 0 \\
0 & 1 & 0 & 0 \\
c & 0 & d & 0 \\
0 & 0 & 0 & 1
\end{array}\right) \times\left(\begin{array}{cccc}
1_{n} & 0 & 0 & \mu \\
{ }^{t} \lambda & 1 & { }^{t} \mu & \kappa \\
0 & 0 & 1_{n} & -\lambda \\
0 & 0 & 0 & 1
\end{array}\right) ;\left(\begin{array}{cc}
a & b \\
c & d
\end{array}\right) \in \Gamma_{n}, \lambda, \mu \in \mathbb{Z}^{n}, \kappa \in \mathbb{Z}\right\} .
$$

We write an element of $H_{n+1}$ as $\left(\begin{array}{cc}\tau & z \\ t z & \omega\end{array}\right)$ where $(\tau, z) \in H_{n} \times \mathbb{C}^{n}$ and $\omega \in H_{1}$. For any integer $m$ and a complex number $x$, we write $e(x)=\exp (2 \pi i x)$ and $e^{m}(x)=e(m x)$. For any $\gamma \in \Gamma_{n}^{J}$ and a holomorphic function $F(\tau, z)$ on $H_{n} \times \mathbb{C}^{n}$, we have $\left.\left(F(\tau, z) e^{m}(\omega)\right)\right|_{k}[\gamma]=\widetilde{F}(\tau, z) e^{m}(\omega)$ for a certain holomorphic function $\widetilde{F}$ on $H_{n} \times \mathbb{C}^{n}$ uniquely determined by $F$ and $\gamma$. Then the mapping $F \mapsto \widetilde{F}$ determines an action of $\Gamma_{n}^{J}$ and we write $\widetilde{F}=\left.F\right|_{k, m}[\gamma]$. When $n \geq 2$, we say that a holomorphic function $F$ on $H_{n} \times \mathbb{C}^{n}$ is a Jacobi form of weight $k$ of index $m$ with respect to $\Gamma_{n}^{J}$ if $\left.F\right|_{k, m}[\gamma]=F$ for any $\gamma \in \Gamma_{n}^{J}$. By the periodicity coming from automorphy, any Jacobi form $F(\tau, z)$ has the Fourier expansion

$$
F(\tau, z)=\sum_{N, r} a(N, r) e\left(\operatorname{Tr}(N \tau)+{ }^{t} r z\right)
$$

where $N$ runs over half-integral symmetric matrices and $r$ over $\mathbb{Z}^{n}$. We have $a(N, r)=0$ unless $4 N m-r^{t} r \geq 0$ (positive semi-definite) by the Koecher principle for $n \geq 2$ proved by Ziegler [23]. When $n=1$, this condition is not automatically satisfied and we add this condition as part of the definition of Jacobi forms. Here note that $r$ is a column vector, so $r^{t} r$ is an $n \times n$ matrix. We say that $F$ is a Jacobi cusp form when $a(N, r)=0$ unless $4 N m-r^{t} r>0$ (positive definite). We denote by $J_{k, m}\left(\Gamma_{n}^{J}\right)$ the space of Jacobi forms defined above, and by $J_{k, m}^{\text {cusp }}\left(\Gamma_{n}^{J}\right)$ the space of Jacobi cusp forms. We note that if $m>0$, then $J_{0, m}\left(\Gamma_{n}^{J}\right)=0$.

\section{$\S 3$. Taylor expansions}

Since a Jacobi form $F(\tau, z)$ is a holomorphic function, we have the Taylor expansion at $z=0$. We write this expansion as

$$
F(\tau, z)=\sum_{\nu=0}^{\infty}\left(\sum_{|\alpha|=\nu} f_{\alpha}(\tau) z^{\alpha}\right),
$$

where $\alpha \in\left(\mathbb{Z}_{\geq 0}\right)^{n}$. We also write $f_{\nu}(\tau, z)=\sum_{|\alpha|=\nu} f_{\alpha}(\tau) z^{\alpha}$. The coefficients $f_{\alpha}(\tau)$ are holomorphic functions on $H_{n}$. They are closely related to Siegel modular forms of degree $n$ as we shall see later. When $n=1$, Eichler-Zagier proved the following results (cf. [5]). 
EZ 1. We put $\epsilon=0$ or 1 if $k$ is even or odd, respectively. For each integer $\nu \geq 0$ with $\nu \equiv k \bmod 2$, we can construct a modular form $\xi_{k+\nu}(\tau) \in M_{k+\nu}\left(\Gamma_{1}\right)$ from the Taylor coefficients $\left(f_{\epsilon}(\tau), f_{\epsilon+2}(\tau), \ldots, f_{\nu}(\tau)\right)$ of a Jacobi form in $J_{k, m}\left(\Gamma_{1}^{J}\right)$. This is explicitly given by using differential operators on $f_{l}(\tau)$ with respect to the variable $\tau$.

EZ 2. When $k$ is even, the linear mapping

$$
J_{k, m}\left(\Gamma_{1}^{J}\right) \rightarrow M_{k}\left(\Gamma_{1}\right) \times M_{k+2}\left(\Gamma_{1}\right) \times \cdots \times M_{k+2 m}\left(\Gamma_{1}\right)
$$

induced by the above construction is injective. In other words, the Jacobi form $F$ is determined by the Taylor coefficients up to $z^{2 m}$. When $k$ is odd, it is determined only by those up to $2 m-3$.

EZ 3. When $m=1$, the above mapping induces a surjective isomorphism from $J_{k, 1}\left(\Gamma_{1}^{J}\right)$ to $M_{k}\left(\Gamma_{1}\right) \oplus S_{k+2}\left(\Gamma_{1}\right)$ for $k>0$.

Now we generalize this to higher $n$. By the action of $-1_{2 n}$ on a Jacobi form $F(\tau, z) \in J_{k, m}\left(\Gamma_{n}^{J}\right)$, we see $F(\tau,-z)=(-1)^{n k} F(\tau, z)$. So $F(\tau, z)$ is an even or odd function of $z$ if $n k$ is even or odd, respectively. To make the notation simpler, we put $\epsilon=0$ or 1 according to whether $n k$ is even or odd. So the Taylor expansion is written as

$$
F(\tau, z)=\sum_{l=0}^{\infty} f_{2 l+\epsilon}(\tau, z) .
$$

We denote by $u={ }^{t}\left(u_{1}, \ldots, u_{n}\right)$ a variable column vector of length $n$. We denote by $\mathcal{H}$ the ring of holomorphic functions on $H_{n}$. Let $\mathcal{H}[u]$ be the polynomial ring in $n$ variables $u_{1}, \ldots, u_{n}$ over $\mathcal{H}$ and $\mathcal{H}[u]_{\nu}$ the vector space of homogeneous polynomials in $\mathcal{H}[u]$ of degree $\nu$. We define a differential operator $\mathcal{D}_{2}$ from $\mathcal{H}[u]_{\nu}$ to $\mathcal{H}[u]_{\nu+2}$ by

$$
\mathcal{D}_{2}={ }^{t} u\left(\frac{1+\delta_{i j}}{2} \frac{\partial}{\partial \tau_{i j}}\right) u=\sum_{i \leq j} u_{i} u_{j} \frac{\partial}{\partial \tau_{i j}},
$$

where $\delta_{i j}$ is Kronecker's delta and $\tau=\left(\tau_{i j}\right) \in H_{n}$. For any non-negative integer $\nu \equiv n k \bmod 2$ and the Taylor coefficients $f_{\nu-2 \mu}(\tau, z)$ of $F(\tau, z)$ with $2 \mu \leq \nu$, which are polynomials in $z$, we define $\xi_{k, \nu}(\tau, u) \in \mathcal{H}[u]_{\nu}$ by

$$
\begin{aligned}
\xi_{k, \nu}(\tau, u) & =\sum_{\mu=0}^{[\nu / 2]} \frac{(k+\nu-\mu-2) !}{\mu !(k+\nu-2) !}(-2 \pi i m)^{\mu}\left(\mathcal{D}_{2}^{\mu} f_{\nu-2 \mu}\right)(\tau, u) \\
& =f_{\nu}(\tau, u)+\text { constant times derivatives of } f_{\nu-2 \mu}(\tau, u) \text { with } \mu>0
\end{aligned}
$$

When $\nu=0$, this does not depend on $u$ so we sometimes write $\xi_{k, 0}(\tau, u)=\xi_{k, 0}(\tau)$. 
By induction, we can show conversely that

$$
f_{\nu}(\tau, u)=\sum_{\mu=0}^{[\nu / 2]} \frac{(2 \pi i m)^{\mu}(k+\nu-2 \mu-1) !}{(k+\nu-\mu-1) ! \mu !} \mathcal{D}_{2}^{\mu} \xi_{k, \nu-2 \mu}(\tau, u) .
$$

Theorem 3.1. Fix a natural number $m$. For any non-negative integer $\nu$ with $\nu \equiv n k \bmod 2$ and any Jacobi form $F \in J_{k, m}\left(\Gamma_{n}^{J}\right)$, define $\xi_{k, \nu}(\tau, u)$ as above. Then $\xi_{k, \nu}(\tau, u) \in A_{k, \nu}\left(\Gamma_{n}\right)$. If $F \in J_{k, m}^{\text {cusp }}\left(\Gamma_{2}^{J}\right)$, then $\xi_{k, \nu}(\tau, u) \in S_{k, \nu}\left(\Gamma_{2}\right)$. The Taylor coefficients $f_{\nu-2 \mu}(\tau, u)$ of $F$ with $2 \mu \leq \nu$ are uniquely determined by $\xi_{k, \nu-2 \mu}(\tau, u)$ with $2 \mu \leq \nu$.

Concrete examples of $\xi_{k, \nu}(\tau, u)$ are as follows:

$$
\begin{aligned}
& \xi_{k, 0}(\tau, u)=f_{0}(\tau) \\
& \xi_{k, 2}(\tau, u)=\sum_{|\alpha|=2} f_{\alpha}(\tau) u^{\alpha}-\frac{2 \pi i m}{k} \sum_{1 \leq i \leq j \leq n} \frac{\partial f_{0}(\tau)}{\partial \tau_{i j}} u_{i} u_{j} .
\end{aligned}
$$

To make it readable, we give a concrete shape of $\xi_{k, 4}(\tau, u)$ only in the case $n=2$. In this case we have

$$
\begin{aligned}
\xi_{k, 4}(\tau, u)= & \left(f_{40}(\tau) u_{1}^{4}+f_{31}(\tau) u_{1}^{3} u_{2}+f_{22}(\tau) u_{1}^{2} u_{2}^{2}+f_{13}(\tau) u_{1} u_{2}^{3}+f_{04}(\tau) u_{2}^{4}\right) \\
& -\frac{2 \pi i m}{k+2}\left(\frac{\partial f_{20}(\tau)}{\partial \tau_{1}} u_{1}^{4}+\left(\frac{\partial f_{20}(\tau)}{\partial z_{0}}+\frac{\partial f_{11}(\tau)}{\partial \tau_{1}}\right) u_{1}^{3} u_{2}\right. \\
& +\left(\frac{\partial f_{20}(\tau)}{\partial \tau_{2}}+\frac{\partial f_{11}(\tau)}{\partial z_{0}}+\frac{\partial f_{02}(\tau)}{\partial \tau_{1}}\right) u_{1}^{2} u_{2}^{2} \\
& \left.+\left(\frac{\partial f_{11}(\tau)}{\partial \tau_{2}}+\frac{\partial f_{02}(\tau)}{\partial z_{0}}\right) u_{1} u_{2}^{3}+\frac{\partial f_{02}(\tau)}{\partial \tau_{2}} u_{2}^{4}\right) \\
& +\frac{(2 \pi i m)^{2}}{2(k+2)(k+1)}\left(\frac{\partial^{2} f_{0}(\tau)}{\partial \tau_{1}^{2}} u_{1}^{4}+2 \frac{\partial^{2} f_{0}(\tau)}{\partial \tau_{1} \partial z_{0}} u_{1}^{3} u_{2}\right. \\
& \left.+\left(\frac{\partial^{2} f_{0}(\tau)}{\partial z_{0}^{2}}+2 \frac{\partial^{2} f_{0}(\tau)}{\partial \tau_{1} \partial \tau_{2}}\right) u_{1}^{2} u_{2}^{2}+2 \frac{\partial^{2} f_{0}(\tau)}{\partial z_{0} \partial \tau_{2}} u_{1} u_{2}^{3}+\frac{\partial^{2} f_{0}(\tau)}{\partial \tau_{2}^{2}} u_{2}^{4}\right),
\end{aligned}
$$

where we write

$$
F(\tau, z)=f_{0}(\tau)+f_{20}(\tau) z_{1}^{2}+f_{11}(\tau) z_{1} z_{2}+f_{02}(\tau) z_{2}^{2}+f_{40}(\tau) z_{1}^{4}+\cdots .
$$

We put $\epsilon=0$ or 1 for $n k \equiv 0$ or $1 \bmod 2$. Then by the above theorem, for any $\nu \in \mathbb{Z}_{\geq \epsilon}$ with $\nu \equiv n k \bmod 2,\left(\xi_{k, 2 \mu+\epsilon}(\tau, u)\right)_{2 \mu \leq \nu}$ induces a linear mapping from $J_{k, m}\left(\Gamma_{n}^{J}\right)$ to $A_{k, \epsilon}\left(\Gamma_{n}\right) \times A_{k, \epsilon+2}\left(\Gamma_{n}\right) \times \cdots \times A_{k, \nu}\left(\Gamma_{n}\right)$.

Theorem 3.1 is a generalization of the case $n=1$ of [5] since when $n=1$ we have $\operatorname{det}^{k} \operatorname{Sym}_{2 l+\epsilon}=\operatorname{det}^{k+2 l+\epsilon}$. Note that when $n=1, \xi_{k, \nu}$ for $\nu>0$ is always a cusp form, but this is not true when $n>1$. There is also the following difference for general $n$. When $n=1$ and when $k$ is even for example, the induced mapping 
from $J_{k, m}\left(\Gamma_{1}\right)$ to $A_{k}\left(\Gamma_{1}\right) \times A_{k+2}\left(\Gamma_{2}\right) \times \cdots \times A_{k, 2 m}\left(\Gamma_{1}\right)$ is injective. But this is not true for general $n$. In fact, there exist non-zero Jacobi forms for $n=m=2$ whose Taylor coefficients vanish up to degree $2 m=4$, as we will see later. It does not seem to be known exactly how many vanishing Taylor coefficients of $F(\tau, z)$ ensure $F(\tau, z)=0$ in general, and this seems an interesting question. (There are several algebro-geometric results for each fixed $\tau$ but they do not fit into our setting of modular forms.)

Proof of Theorem 3.1. It is possible to prove Theorem 3.1 by direct calculation, but here we use differential operators on Siegel modular forms of degree $n+1$. A general theory of holomorphic differential operators on Siegel modular forms which behave well under restriction of the domain is given in [9]. So before proving the above theorem, we recall part of the results in [9] which will be used in the proof. We fix non-negative integers $k$ and $l$. For any $g_{1}=\left(\begin{array}{ll}a_{1} & b_{1} \\ c_{1} & d_{1}\end{array}\right) \in S p(n, \mathbb{R})$ and $g_{2}=\left(\begin{array}{ll}a_{2} & b_{2} \\ c_{2} & d_{2}\end{array}\right) \in S L(2, \mathbb{R})$ and any $V_{\nu}$-valued function $f$ of $(\tau, \omega) \in H_{n} \times H_{1}$, we write

$$
\left.f(\tau, \omega)\right|_{k, \nu}\left[\left(g_{1}, g_{2}\right)\right]=\left(c_{2} \omega+d_{2}\right)^{-k-\nu} \rho_{k, \nu}\left(c_{1} \tau+d_{1}\right)^{-1} f\left(g_{1} \tau, g_{2} \omega\right) .
$$

This is an action of $S p(n, \mathbb{R}) \times S L(2, \mathbb{R})$. We define a group embedding of $S p(n, \mathbb{R}) \times$ $S L(2, \mathbb{R})$ into $S p(n+1, \mathbb{R})$ by

$$
\iota\left(g_{1}, g_{2}\right)=\left(\begin{array}{cccc}
a_{1} & 0 & b_{1} & 0 \\
0 & a_{2} & 0 & b_{2} \\
c_{1} & 0 & d_{1} & 0 \\
0 & c_{2} & 0 & d_{2}
\end{array}\right) .
$$

For $Z \in H_{n+1}$ we write $Z=\left(\begin{array}{cc}\tau & z \\ t & \omega\end{array}\right)$. For any function $G$ on $H_{n+1}$, we denote by Res the following restriction:

$$
(\operatorname{Res} G)(\tau, \omega)=G\left(\begin{array}{ll}
\tau & 0 \\
0 & \omega
\end{array}\right)
$$

To define our operator, we use Gegenbauer polynomials. For any non-negative integer $\nu$ and $k \geq 2$, we define polynomials $P_{\nu}^{(k)}(s, w)$ in two variables $s$ and $w$ by the following formal power series of $t$ :

$$
\frac{1}{\left(1-2 s t+w t^{2}\right)^{k-1}}=\sum_{\nu=0}^{\infty} P_{\nu}^{(k)}(s, w) t^{\nu}
$$

Then we have

$$
P_{\nu}^{(k)}(s, m)=\sum_{\mu=0}^{[\nu / 2]}(-1)^{\mu} \frac{(k+\nu-\mu-2) !}{(k-2) !(\nu-2 \mu) ! \mu !}(2 s)^{\nu-2 \mu} w^{\mu} .
$$


The polynomials $P_{\nu}^{(k)}(s, 1)$ are classical Gegenbauer polynomials. We define a $V_{\nu^{-}}$ valued differential operator by

$$
\mathbb{D}_{k, \nu}=P_{\nu}^{(k)}\left(\frac{1}{2} \sum_{i=1}^{n} u_{i} \frac{\partial}{\partial z_{i}}, \frac{\partial}{\partial \omega} \mathcal{D}_{2}\right) .
$$

Then for any holomorphic function $G(Z)$ on $H_{n+1}$ and $\left(g_{1}, g_{2}\right) \in S p(n, \mathbb{R}) \times$ $S L(2, \mathbb{R})$, we have

$$
\operatorname{Res}\left(\mathbb{D}_{k, \nu}\left(\left.G\right|_{k} \iota\left(g_{1}, g_{2}\right)\right)\right)=\left.\left(\operatorname{Res}\left(\mathbb{D}_{k, \nu} G\right)\right)\right|_{k, \nu}\left(g_{1}, g_{2}\right) .
$$

Actually a linear differential operator with constant coefficients which satisfies this relation is unique up to a constant if $2 k \geq n$. For the proof, see [9, p. 114].

Now we take a holomorphic function $F(\tau, z)$ on $H_{n} \times \mathbb{C}^{n}$ and put $G(Z)=$ $F(\tau, z) e^{m}(\omega)$. For any $g \in S p(n, \mathbb{R})$, we write the Taylor expansion of $\left.F\right|_{k, m}[g]$ at $z=0$ as $\left(\left.F\right|_{k, m}[g]\right)(\tau, z)=\sum_{\nu=0}^{\infty} f_{\nu, g}(\tau, z)$, where $f_{\nu, g}(\tau, z)$ is the homogeneous terms in $z$ of total degree $\nu$. For a fixed $F$ and $g$, we put

$$
\xi_{k, \nu, g}(\tau, \nu)=\sum_{\mu=0}^{[\nu / 2]}(-1)^{\mu}(-2 \pi i m)^{\mu} \frac{(k+\nu-\mu-2) !}{\mu !(k+\nu-2) !} \mathcal{D}_{2}^{\mu} f_{\nu-2 \mu, g}(\tau, u) .
$$

In particular, by definition we have $\xi_{k, \nu}(\tau, u)=\xi_{k, \nu, 1_{2 n}}(\tau, u)$. For any $\alpha \in\left(\mathbb{Z}_{\geq 0}\right)^{n}$ with $|\alpha|=\nu-2 \mu$, we have

$$
\left(\sum_{i=1}^{n} u_{i} \frac{\partial}{\partial z_{i}}\right)^{\nu-2 \mu} z^{\alpha}=(\nu-2 \mu) ! u^{\alpha},
$$

so calculating directly from the definition, we have

$$
\frac{(k-2) !}{(k+\nu-2) !} \operatorname{Res}\left(\mathbb{D}_{k, \nu}\left(\left.G\right|_{k} \iota\left(g, 1_{2}\right)\right)\right)=\xi_{k, \nu, g}(\tau, u) e^{m}(\omega) .
$$

Since this is equal to $\left.\left(\operatorname{Res}\left(\mathbb{D}_{k, \nu} G\right)\right)\right|_{k, \nu}\left[\left(g, 1_{2}\right)\right] \times(k-2) ! /(k+\nu-2)$ !, we have

$$
\xi_{k, \nu, g}(\tau, u)=\left.\xi_{k, \nu}(\tau, u)\right|_{k, \nu}[g] .
$$

In particular, if $F \in J_{k, m}\left(\Gamma_{n}^{J}\right)$, then $\left.F\right|_{k, m}[\gamma]=F$ for any $\gamma \in \Gamma_{n}$ and $\xi_{k, \nu, \gamma}=$ $\xi_{k, \nu}$. So $\left.\xi_{k, \nu}\right|_{k, \nu}[\gamma]=\xi_{k, \nu}$ and $\xi_{k, \nu}(\tau, u) \in A_{k, \nu}\left(\Gamma_{2}\right)$. If $F \in J_{k, m}^{\text {cusp }}\left(\Gamma_{n}^{J}\right)$, then by definition, the Fourier coefficient $a(N, r)$ of $F(\tau, z)$ vanishes unless $4 m N-r^{t} r>0$. So it vanishes unless $N>0$, and since the Fourier coefficients of $\xi_{k, \nu}$ are linear combinations of these $a(N, r)$, the same holds for $\xi_{k, \nu}(\tau, u)$, which means that $\xi_{k, \nu} \in S_{k, \nu}\left(\Gamma_{n}\right)$.

The coefficients of the differential operator are rational functions of $k$ well defined for $k \geq 1$ and since the operator is determined algebraically with respect to $k$, this proves Theorem 3.1 for any $k \geq 1$. 
By the way, the normalization of $\xi_{k, \nu}$ is consistent with [5] when $n=1$. If a more direct approach is preferred, one can use the following formulas:

$$
\begin{aligned}
\mathcal{D}_{2}(\operatorname{det}(c \tau+d)) & =\operatorname{det}(c \tau+d)\left({ }^{t} u(c \tau+d)^{-1} c u\right), \\
\mathcal{D}_{2}\left(\operatorname{det}(c \tau+d)^{k}\right) & =k \operatorname{det}(c \tau+d)^{k}\left({ }^{t} u(c \tau+d)^{-1} c u\right), \\
\mathcal{D}_{2}\left(\left({ }^{t}(c \tau+d)^{-1} u\right)^{\alpha}\right) & =-|\alpha|\left({ }^{t} u(c \tau+d)^{-1} c u\right)\left({ }^{t}(c \tau+d)^{-1} u\right)^{\alpha}, \\
\mathcal{D}_{2}\left(\left({ }^{t} u(c \tau+d)^{-1} c u\right)^{\mu}\right) & =-\mu\left({ }^{t} u(c \tau+d)^{-1} c u\right)^{\mu+1} .
\end{aligned}
$$

Here $\mu$ is any integer and $\alpha$ is a multi-index. For any $f(\tau, u) \in \mathcal{H}[u]_{\nu}$ and $g=$ $\left(\begin{array}{ll}a & b \\ c & d\end{array}\right) \in S p(n, \mathbb{R})$, we have

$$
\begin{aligned}
& \mathcal{D}_{2}\left(f\left(g \tau,{ }^{t}(c \tau+d)^{-1} u\right)\right) \\
& \quad=-\nu\left({ }^{t} u(c \tau+d)^{-1} c u\right) f\left(g \tau,{ }^{t}(c \tau+d)^{-1} u\right)+\left(\mathcal{D}_{2} f\right)\left(g \tau,{ }^{t}(c \tau+d)^{-1} u\right) .
\end{aligned}
$$

These formulas can be obtained by standard matrix calculations. Also by applying these to the Taylor expansion of Jacobi forms, we obtain an alternative proof of Theorem 3.1. We omit the details here.

\section{$\S 4$. Theta expansions and transformation formulas}

Now we explain another expansion of $F(\tau, z)$ which we call "theta expansion". This will be used in later sections. For any $m \in \mathbb{Z}_{>0}$, any Jacobi form $F \in J_{k, m}\left(\Gamma_{n}^{J}\right)$ satisfies

$$
F(\tau, z+\tau \lambda+\mu)=e^{m}\left(-{ }^{t} \lambda \tau \lambda-2{ }^{t} \lambda z\right) F(\tau, z)
$$

for any $\lambda, \mu \in \mathbb{Z}^{n}$. For any $\nu \in \mathbb{Z}^{n}$, we put

$$
\vartheta_{\nu, m}(\tau, z)=\sum_{p \in \mathbb{Z}^{n}} e\left({ }^{t}\left(p+\frac{\nu}{2 m}\right)(m \tau)\left(p+\frac{\nu}{2 m}\right)+\left(p+\frac{\nu}{2 m}\right)(2 m z)\right) .
$$

This series depends only on $\nu \bmod 2 m$, and there are $(2 m)^{n}$ linearly independent such functions. Then by the well-known theory of theta functions, any function on $H_{n} \times \mathbb{C}^{n}$ which satisfies (4.1) is a linear combination of these theta functions as a function of $z$. In particular, for Jacobi forms $F$ we have

$$
F(\tau, z)=\sum_{\nu \in(\mathbb{Z} / 2 m \mathbb{Z})^{n}} c_{\nu}(\tau) \vartheta_{\nu, m}(\tau, z)
$$

for some holomorphic functions $c_{\nu}(\tau)$ on $H_{n}$. But $F$ is automorphic also for $\Gamma_{n}$, so we can say a little more. By the action of $-1_{2 n} \in \Gamma_{n}$, we have $F(\tau,-z)=$ $(-1)^{n k} F(\tau, z)$, so for example if $n k$ is even, then $F(\tau, z)$ is an even function of $z$. 
But we also have $\vartheta_{\nu, m}(\tau,-z)=\vartheta_{-\nu, m}(\tau, z)$, so this means that $c_{\nu}(\tau)=c_{-\nu}(\tau)$. If $m=1$, this does not give any new condition, since $-\nu \equiv \nu \bmod 2$ and theta functions $\vartheta_{\nu, 1}(\tau, z)$ are all even functions of $z$. But when $m>1$, the above relation gives a real restriction, as we will see in Section 5 .

Since we will sometimes use the theta transformation formulas, we recall them here. We write $\vartheta_{\nu, m}(\tau)=\vartheta_{\nu, m}(\tau, 0)$. For any $U \in G L_{n}(\mathbb{R}), S={ }^{t} S \in M_{n}(\mathbb{R})$, we write

$$
t(U)=\left(\begin{array}{cc}
U & 0 \\
0 & { }^{t} U^{-1}
\end{array}\right), \quad u(S)=\left(\begin{array}{cc}
1_{n} & S \\
0 & 1_{n}
\end{array}\right) .
$$

Then $\Gamma_{n}$ is generated by $t(U)\left(U \in G L_{n}(\mathbb{Z})\right), u(S)\left(S={ }^{t} S \in M_{n}(\mathbb{Z})\right)$, and $J_{n}$. By definition, we have

$$
\begin{aligned}
\vartheta_{\nu, m}(\tau+S, z) & =e\left({ }^{t} \nu S \nu / 4 m\right) \vartheta_{\nu, m}(\tau, z), \\
\vartheta_{\nu, m}\left(U \tau{ }^{t} U,{ }^{t} U z\right) & =\vartheta_{U \nu, m}(\tau, z) .
\end{aligned}
$$

As for $J_{n}$, we need the well known theta transformation formula (cf. e.g. [16, II, p. 226]). For any $\boldsymbol{m}={ }^{t}\left(m^{\prime}, m^{\prime \prime}\right) \in \mathbb{Q}^{n}$, define

$$
\theta_{\boldsymbol{m}}(\tau, z)=\sum_{p \in \mathbb{Z}^{n}} e\left(\frac{1}{2}^{t}\left(p+m^{\prime}\right) \tau\left(p+m^{\prime}\right)+{ }^{t}\left(p+m^{\prime}\right)\left(z+m^{\prime \prime}\right)\right) .
$$

Here $\boldsymbol{m}$ is called a theta characteristic. Then for any $\gamma \in \Gamma_{n}$, we have

$$
\left.\theta_{\gamma \cdot \boldsymbol{m}}(\tau, z)=\kappa(\gamma) e\left(\phi_{\boldsymbol{m}}(\gamma)\right) \operatorname{det}(c \tau+d)^{1 / 2} e\left({ }^{t} z(c \tau+d)^{-1} c z\right) / 2\right) \theta_{\boldsymbol{m}}(\tau, z)
$$

where $\kappa(\gamma)$ is a root of unity, $\gamma \cdot \boldsymbol{m}$ is an action of $\Gamma_{n}$ on $(\mathbb{Q} / \mathbb{Z})^{2 n}$ (which is not just matrix multiplication), $\phi_{\boldsymbol{m}}(\gamma)$ is an explicit function of $\boldsymbol{m}$ and $\gamma$, and $\operatorname{det}(c \tau+d)^{1 / 2}$ is a fixed branch determined by $\tau$ and $\gamma$ on which $\kappa(\gamma)$ depends. We do not recall the precise formula here, but in our application in this paper, we need not specify the branch since it appears always with $\kappa(\gamma)$ and the final results never depend on the choice. Now we have

$$
\begin{aligned}
\vartheta_{\nu, m}\left(-\tau^{-1}, \tau^{-1} z\right) & =\theta_{(\nu / 2 m, 0)}\left(-2 m \tau^{-1}, 2 m \tau^{-1} z\right) \\
& =\theta_{(\nu / 2 m, 0)}\left(-(\tau / 2 m)^{-1},(\tau / 2 m)^{-1} z\right) .
\end{aligned}
$$

If we apply (4.2) for $\gamma=J_{n}$ and $\boldsymbol{m}=(\nu / 2 m, 0)$, then since $J \cdot{ }^{t}(0,-\nu / 2 m)=$ ${ }^{t}(\nu / 2 m, 0)$ and $\phi_{(0,-\nu / 2 m)}\left(J_{n}\right)=0$, we have

$$
\theta_{\nu / 2 m, 0}\left(-\tau^{-1}, \tau^{-1} z\right)=\kappa\left(J_{n}\right) \operatorname{det}(\tau)^{1 / 2} e\left({ }^{t} z \tau^{-1} z / 2\right) \theta_{0,-\nu / 2 m}(\tau, z) .
$$

So

$$
\vartheta_{\nu, m}\left(\tau^{-1}, \tau^{-1} z\right)=\kappa\left(J_{n}\right) \operatorname{det}(\tau / 2 m)^{1 / 2} e^{m}\left({ }^{t} z \tau^{-1} z\right) \theta_{0,-\nu / 2 m}(\tau / 2 m, z) .
$$


But we can rewrite the summation of the definition as

$$
\begin{aligned}
\theta_{0,-\nu / 2 m} & (\tau / 2 m, z)=\sum_{p \in \mathbb{Z}^{n}} e\left({ }^{t} p(\tau / 2 m) p / 2+{ }^{t} p(z-\nu / 2 m)\right) \\
= & \sum_{r \in(\mathbb{Z} / 2 m \mathbb{Z})^{n}} \sum_{l \in \mathbb{Z}^{n}} e\left({ }^{t}(2 m l+r)(\tau / 2 m)(2 m l+r)+{ }^{t}(2 m l+r)(z-\nu / 2 m)\right) \\
= & \sum_{r \in(\mathbb{Z} / 2 m \mathbb{Z})^{n}} e\left(-{ }^{t} r \nu / 2 m\right) \vartheta_{r, m}(\tau, z) .
\end{aligned}
$$

Hence

$$
\begin{aligned}
\vartheta_{\nu, m}( & \left.-\tau^{-1}, \tau^{-1} z\right) \\
& =\kappa\left(J_{n}\right) \operatorname{det}(\tau / 2 m)^{1 / 2} e^{m}\left({ }^{t} z \tau^{-1} z\right) \sum_{r \in(\mathbb{Z} / 2 m \mathbb{Z})^{n}} e\left(-{ }^{t} r \nu / 2 m\right) \vartheta_{r, m}(\tau, z) .
\end{aligned}
$$

Also $\kappa\left(J_{n}\right)^{2}=(-i)^{n}$. This seems more or less known and easily proved also by restricting the transformation formula for $\theta_{\mathbf{0}}(\tau)$ to the diagonals of $\tau$ and using the Poisson formula for $n=1$.

We shall apply the above formula to establish the behaviour of Jacobi forms under some differential operators later.

\section{§5. Explicit structures for index one}

From now on, we treat the case $n=2$. We define the ring of Siegel modular forms by

$$
A\left(\Gamma_{2}\right)=\bigoplus_{k=0}^{\infty} A_{k}\left(\Gamma_{2}\right) \quad \text { and } \quad A_{\text {even }}\left(\Gamma_{2}\right)=\bigoplus_{k=0}^{\infty} A_{2 k}\left(\Gamma_{2}\right) .
$$

It is well known from Igusa [15] that $A_{\text {even }}\left(\Gamma_{2}\right)$ is generated by Siegel modular forms $\phi_{4}, \phi_{6}, \chi_{10}, \chi_{12}$ and for $A\left(\Gamma_{2}\right)$ we moreover need $\chi_{35}$, where each subscript is the weight of the form. For any fixed natural number $m$, we write $J_{m}\left(\Gamma_{2}^{J}\right)=\bigoplus_{k>0}^{\infty} J_{k, m}\left(\Gamma_{2}^{J}\right), J_{\text {even,m }}\left(\Gamma_{2}^{J}\right)=\bigoplus_{k>0} J_{2 k, m}\left(\Gamma_{2}^{J}\right)$, and $J_{\text {odd }, m}\left(\Gamma_{2}^{J}\right)=$ $\bigoplus_{k=0}^{\infty} J_{2 k+1, m}\left(\Gamma_{2}^{J}\right)$. These modules are obviously $A_{\text {even }}\left(\Gamma_{2}\right)$-modules and the first one is also an $A\left(\Gamma_{2}\right)$-module. We would like to study the structure of these modules only over $A_{\text {even }}\left(\Gamma_{2}\right)$ since it becomes rather complicated if we regard it as a module over $A\left(\Gamma_{2}\right)$.

First we give a result for $n=2$ and $m=1$. When $k$ is odd, we have $A_{k, j}\left(\Gamma_{2}\right)=$ $S_{k, j}\left(\Gamma_{2}\right)$ for any $j \geq 0$. For odd $k$, we put

$$
S_{k, 2}^{0}\left(\Gamma_{2}\right)=\left\{f(\tau, u) \in A_{k, 2}\left(\Gamma_{2}\right) ; f\left(\left(\begin{array}{cc}
\tau_{1} & 0 \\
0 & \tau_{2}
\end{array}\right), u\right)=0\right\}
$$


where we write $\tau=\left(\begin{array}{cc}\tau_{1} & z_{0} \\ z_{0} & \tau_{2}\end{array}\right) \in H_{2}$. Note that explicit structures of $A_{k, 2}\left(\Gamma_{2}\right)$ and $S_{k, 2}^{0}\left(\Gamma_{2}\right)$ are known (see [12], [10]).

We note that if we define $S_{k}^{0}\left(\Gamma_{2}\right)$ in the same way for odd $k$, then it is well known that $S_{k}\left(\Gamma_{2}\right)=S_{k}^{0}\left(\Gamma_{2}\right)$, so this is redundant.

Theorem 5.1. Assume that $n=2$.

(1) For any natural number $k$, the mapping

$$
J_{k, 1}\left(\Gamma_{2}^{J}\right) \ni F \mapsto\left(\xi_{k, 0}(\tau), \xi_{k, 2}(\tau, u)\right) \in A_{k}\left(\Gamma_{2}\right) \times A_{k, 2}\left(\Gamma_{2}\right)
$$

is injective.

(2) If $k$ is even with $k \geq 2$, this mapping is also surjective.

(3) If $k$ is odd, then the image of the mapping in (1) is $S_{k}\left(\Gamma_{2}\right) \times S_{k, 2}^{0}\left(\Gamma_{2}\right)$.

(4) $J_{1}\left(\Gamma_{2}^{J}\right)$ is a free $A_{\mathrm{even}}\left(\Gamma_{2}\right)$-module spanned by Jacobi forms of respective weights $4,6,10,12,21,27,29,35$.

Assertion (4) of this theorem is essentially contained in [7]. The proof there used structures of the "plus" space (a kind of space of new forms) of Siegel modular forms of half-integral weight of level 4 with or without character, since $J_{k, 1}\left(\Gamma_{2}^{J}\right)$ is isomorphic to this space (cf. [8], [7]). But there we needed a complicated calculation to extract the plus space from the whole space of half-integral weight. In this paper, we give a more direct simple proof.

Before proving the theorem, we first show some transformation formula for theta series. We consider a differential operator on four holomorphic functions $F_{i}(\tau)(i=1, \ldots, 4)$ on $H_{2}$. We write

$$
(\tau, z)=\left(\left(\begin{array}{cc}
\tau_{1} & z_{0} \\
z_{0} & \tau_{2}
\end{array}\right),\left(\begin{array}{c}
z_{1} \\
z_{2}
\end{array}\right)\right) \in H_{2} \times \mathbb{C}^{2}
$$

and put $\partial_{i}=\frac{1}{2 \pi i} \frac{\partial}{\partial z_{i}}, \partial_{i i}=\frac{1}{2 \pi i} \frac{\partial}{\partial \tau_{i}}$ for $i=1,2$, and $\partial_{12}=\frac{1}{4 \pi i} \frac{\partial}{\partial z_{0}}$. For functions $F_{i}(\tau)(1 \leq i \leq 4)$ of $\tau \in H_{2}$, we define

$$
\left\{F_{1}, F_{2}, F_{3}, F_{4}\right\}_{3}=\left|\begin{array}{cccc}
F_{1} & F_{2} & F_{3} & F_{4} \\
\partial_{11} F_{1} & \partial_{11} F_{2} & \partial_{11} F_{3} & \partial_{11} F_{4} \\
\partial_{12} F_{1} & \partial_{12} F_{2} & \partial_{12} F_{3} & \partial_{12} F_{4} \\
\partial_{22} F_{1} & \partial_{22} F_{2} & \partial_{22} F_{3} & \partial_{22} F_{4}
\end{array}\right| .
$$

For any $g=\left(\begin{array}{ll}a & b \\ c & d\end{array}\right) \in S p(2, \mathbb{R})$, we can fix a branch of $\operatorname{det}(c \tau+d)^{1 / 2}$ on $H_{2}$. Although this is not an automorphy factor, it is a well defined function for a fixed $g$ since $H_{2}$ is simply connected (and the branch depends on $\tau$ itself and is not determined by $\operatorname{det}(c \tau+d))$. For any function $F(\tau)$ on $H_{2}$, we define $\left(\left.F\right|_{1 / 2}[g]\right)(\tau)=$ 
$\operatorname{det}(c \tau+d)^{-1 / 2} F(g \tau)$. Then

$$
\left\{\left.F_{1}\right|_{1 / 2}[g],\left.F_{2}\right|_{1 / 2}[g],\left.F_{3}\right|_{1 / 2}[g],\left.F_{4}\right|_{1 / 2}[g]\right\}_{3}=\left.\left\{F_{1}, F_{2}, F_{3}, F_{4}\right\}_{3}\right|_{5}[g]
$$

for any $g \in S p(2, \mathbb{R})$. Here the subscript 3 is used to indicate $4 \cdot 1 / 2+3=5$. This is a special case of the operator in [9, Theorem 2], or [2, p. 251]. Now we apply this to the theta functions for $m=1$. Here in the case of $n=2$ and index $m=1$, for any $\nu \in(\mathbb{Z} / 2 \mathbb{Z})^{2}$, we write $\vartheta_{\nu}(\tau, z)=\vartheta_{\nu, 1}(\tau, z)$ and $\vartheta_{\nu}(\tau)=\vartheta_{\nu}(\tau, 0)$ for simplicity. We show that $\left\{\vartheta_{00}(\tau), \vartheta_{01}(\tau), \vartheta_{10}(\tau), \vartheta_{11}(\tau)\right\}_{3}$ is a Siegel modular form of $\Gamma_{2}$ of weight 5 with a multiplier system. By the formula given in the previous section, we have

$$
\begin{aligned}
\left(\left.\vartheta_{00}(\tau)\right|_{1 / 2}\left[J_{2}\right],\left.\vartheta_{01}(\tau)\right|_{1 / 2}\left[J_{2}\right],\left.\vartheta_{10}(\tau)\right|_{1 / 2}\left[J_{2}\right],\left.\vartheta_{11}(\tau)\right|_{1 / 2}\left[J_{2}\right]\right) & \\
& =\left(\vartheta_{00}(\tau), \vartheta_{01}(\tau), \vartheta_{10}(\tau), \vartheta_{11}(\tau)\right) A
\end{aligned}
$$

where

$$
A=2^{-1} \kappa\left(J_{2}\right)\left(\begin{array}{cccc}
1 & 1 & 1 & 1 \\
1 & -1 & 1 & -1 \\
1 & 1 & -1 & -1 \\
1 & -1 & -1 & 1
\end{array}\right) .
$$

We consider the $4 \times 4$ matrix whose rows are $\left(\vartheta_{00}(\tau), \vartheta_{01}(\tau), \vartheta_{10}(\tau), \vartheta_{11}(\tau)\right) A$ and its $\partial_{11}, \partial_{12}$ and $\partial_{22}$ derivatives. Taking the determinant of this matrix, we see that

$$
\begin{aligned}
\left\{\left.\vartheta_{00}(\tau)\right|_{1 / 2}\left[J_{2}\right],\left.\vartheta_{01}(\tau)\right|_{1 / 2}\left[J_{2}\right],\left.\vartheta_{10}(\tau)\right|_{1 / 2}\left[J_{2}\right],\left.\vartheta_{11}(\tau)\right|_{1 / 2}\left[J_{2}\right]\right\}_{3} \\
=\left\{\vartheta_{00}(\tau), \vartheta_{01}(\tau), \vartheta_{10}(\tau), \vartheta_{11}(\tau)\right\}_{3} \operatorname{det}(A) .
\end{aligned}
$$

Since $\kappa\left(J_{2}\right)^{4}=1$, we have $\operatorname{det}(A)=1$. We put $\chi_{5}=\left\{\vartheta_{00}, \vartheta_{01}, \vartheta_{10}, \vartheta_{11}\right\}_{3}$. Then we can show that this is not identically zero (see e.g. the Fourier coefficients) and by the property of this differential operator explained above, we have $\left.\chi_{5}\right|_{5}\left[J_{2}\right]=\chi_{5}$. As for the action of $t(U)$ and $u(S), t(U)$ for $U \in G L_{2}(\mathbb{Z})$ acts on $\vartheta_{\nu}(\tau)$ as a permutation and the automorphy factor is $\operatorname{det}(U)^{1 / 2 \times 4}=1$, and $u(S)$ for $S=$ $\left(\begin{array}{cc}s_{1} & s_{12} \\ s_{12} & s_{2}\end{array}\right) \in M_{2}(\mathbb{Z})$ acts on $\chi_{5}$ as multiplication by

$$
\sum_{\nu \in(\mathbb{Z} / 2 \mathbb{Z})^{2}} e\left({ }^{t} \nu S \nu / 4\right)=e\left(\left(s_{1}+s_{2}+s_{12}\right) / 2\right) .
$$

The latter is again \pm 1 . So $\left.\chi_{5}\right|_{5} t(U)= \pm \chi_{5}$ and $\chi_{5} \mid u(S)= \pm \chi_{5}$. This means that $\chi_{5}$ is a Siegel modular form of weight 5 of $\Gamma_{2}$ with the multiplier system of $\Gamma_{2}$. Such a Siegel modular form is unique up to a constant and is equal to a constant times $\theta_{0000} \theta_{0001} \theta_{0010} \theta_{0011} \theta_{0100} \theta_{0110} \theta_{1000} \theta_{1001} \theta_{1100} \theta_{1111}$ (cf. [15]). 
Proof of Theorem 5.1. For any $F(\tau, z) \in J_{k, 1}\left(\Gamma_{2}^{J}\right)$, we write

$$
F(\tau, z)=f_{0}(\tau)+\left(f_{20}(\tau) z_{1}^{2}+f_{11}(\tau) z_{1} z_{2}+f_{02}(\tau) z_{2}^{2}\right)+\cdots .
$$

We also use the theta expansion. We have

$$
F(\tau, z)=c_{00}(\tau) \vartheta_{00}(\tau, z)+c_{01}(\tau) \vartheta_{01}(\tau, z)+c_{10}(\tau) \vartheta_{10}(\tau, z)+c_{11}(\tau) \vartheta_{11}(\tau, z)
$$

for some holomorphic functions $c_{\nu}(\tau)$ on $H_{2}$. Here $c_{\nu}(\tau)$ are uniquely determined by $F$. Differentiating both sides with respect to $z_{1}$ and $z_{2}$ at most twice, we have a simultaneous equation

$$
A(\tau) c(\tau)=f(\tau)
$$

where we put

$$
\begin{aligned}
& c(\tau)={ }^{t}\left(c_{00}(\tau), c_{01}(\tau), c_{10}(\tau), c_{11}(\tau)\right), \\
& f(\tau)={ }^{t}\left(f_{0}(\tau), 2 f_{20}(\tau) /(2 \pi i)^{2}, f_{11}(\tau) /(2 \pi i)^{2}, 2 f_{02}(\tau) /(2 \pi i)^{2}\right),
\end{aligned}
$$

and define $A(\tau)$ to be the matrix

$$
\left(\begin{array}{cccc}
\vartheta_{00}(\tau) & \vartheta_{01}(\tau) & \vartheta_{10}(\tau) & \vartheta_{11}(\tau) \\
\left.\partial_{1}^{2} \vartheta_{00}(\tau, z)\right|_{z=0} & \left.\partial_{1}^{2} \vartheta_{01}(\tau, z)\right|_{z=0} & \left.\partial_{1}^{2} \vartheta_{10}(\tau, z)\right|_{z=0} & \left.\partial_{1}^{2} \vartheta_{11}(\tau, z)\right|_{z=0} \\
\left.\partial_{1} \partial_{2} \vartheta_{00}(\tau, z)\right|_{z=0} & \left.\partial_{1} \partial_{2} \vartheta_{01}(\tau, z)\right|_{z=0} & \left.\partial_{1} \partial_{2} \vartheta_{10}(\tau, z)\right|_{z=0} & \left.\partial_{1} \partial_{2} \vartheta_{11}(\tau, z)\right|_{z=0} \\
\left.\partial_{2}^{2} \vartheta_{00}(\tau, z)\right|_{z=0} & \left.\partial_{2}^{2} \vartheta_{01}(\tau, z)\right|_{z=0} & \left.\partial_{2}^{2} \vartheta_{10}(\tau, z)\right|_{z=0} & \left.\partial_{2}^{2} \vartheta_{11}(\tau, z)\right|_{z=0}
\end{array}\right) .
$$

Theta functions satisfy the heat equation $\left.\partial_{i} \partial_{j} \vartheta_{\nu}(\tau, z)\right|_{z=0}=4 \partial_{i j} \vartheta_{\nu}(\tau)$ for any $i, j$ with $1 \leq i, j \leq 2$. (Note that we defined $\partial_{12}=(4 \pi i)^{-1} \frac{\partial}{\partial z_{0}}$ to have a unified relation here.) So $\operatorname{det}(A(\tau))$ is equal to $\chi_{5}(\tau)=\left\{\vartheta_{00}(\tau), \ldots, \vartheta_{11}(\tau)\right\}_{3}$ up to a constant. Here it is well known that $\chi_{5}(\tau)$ vanishes only on the $\Gamma_{2}$-orbit of the diagonals of $H_{2}$ and the vanishing order is one (cf. Freitag [6, p. 145, Hilfssatz 1.3]). Anyway, $\operatorname{det}(A(\tau))$ does not vanish identically, so the mapping from $J_{k, 1}\left(\Gamma_{2}^{J}\right)$ to $A_{k}\left(\Gamma_{2}\right) \times A_{k, 2}\left(\Gamma_{2}\right)$ is injective. When $k$ is even, by comparing the dimensions found by Tsushima [20][22], we can see that the mapping is also surjective. Actually this is proved more directly as follows without using dimension formulas. To show the surjectivity, instead of taking Jacobi forms, we start from some forms $g_{k, 0}(\tau) \in A_{k}\left(\Gamma_{2}\right)$ and $g_{k, 2}(\tau, u) \in A_{k, 2}\left(\Gamma_{2}\right)$. Then we define $f_{\nu}(\tau)$ for $\nu=0,20,11,02$ by (3.2) by putting $\xi_{k, 0}=g_{k, 0}$ and $\xi_{k, 2}=g_{k, 2}$. Then we consider the above simultaneous equation $A(\tau) c(\tau)=f(\tau)$ for this $f(\tau)$, regarding $c_{\nu}(\tau)$ as unknown. Each $\vartheta_{\nu}(\tau)$ is an even function with respect to $z_{0}$. This is shown by replacing $p={ }^{t}\left(p_{1}, p_{2}\right)$ by $\left(p_{1},-p_{2}-\nu_{2}\right)$ in the summation of the definition of each $\vartheta_{\nu}(\tau)$. So each $\partial_{12} \vartheta_{\nu}(\tau)$ is an odd function of $z_{0}$ and hence all the components of the third row of $A(\tau)$ vanish at $z_{0}=0$. So if we denote by $\widetilde{A}(\tau)$ the cofactor matrix of $A(\tau)$, then the first, second and fourth columns are zero at $z_{0}=0$. Now assume that $k$ is 
even. By the action of the diagonal matrix $\operatorname{diag}(1,-1,1,-1) \in \Gamma_{2}$, we see that any form $g(\tau)$ in $A_{k}\left(\Gamma_{2}\right)$ is an even (resp. odd) function of $z_{0}$ if $k$ is even (resp. odd). In particular, $\partial_{12} g$ vanishes at $z_{0}=0$ if $k$ is even. In the same way, if $g_{20}(\tau) u_{1}^{2}+g_{11}(\tau) u_{1} u_{2}+g_{02}(\tau) u_{2}^{2} \in A_{k, 2}\left(\Gamma_{2}\right)$, then we see that $g_{11}(\tau)$ is an odd function of $z_{0}$ if $k$ is even. Since

$$
f_{20}(\tau) u_{1}^{2}+f_{11}(\tau) u_{1} u_{2}+f_{02}(\tau) u_{2}^{2}=\xi_{k, 2}(\tau)+\frac{2 \pi i}{k} \sum_{1 \leq i \leq j \leq 2} \frac{\partial \xi_{k, 0}(\tau)}{\partial \tau_{i j}} u_{i} u_{j}
$$

$f_{11}(\tau)$ also vanishes at $z_{0}=0$. So $A(\tau)^{-1} f(\tau)$ is holomorphic at $z_{0}=0$ when $k$ is even and this means that the unique solution $c(\tau)$ of the simultaneous equation $A(\tau) c(\tau)=f(\tau)$ is holomorphic on the fundamental domain $\mathcal{F}$ of $\Gamma_{2}$. Now we define a function $F(\tau, z)$ on $H_{2} \times \mathbb{C}^{2}$ by $F(\tau, z)=\sum_{\nu \in(\mathbb{Z} / 2 \mathbb{Z})^{2}} c_{\nu}(\tau) \vartheta_{\nu}(\tau, z)$, using the components $c_{\nu}(\tau)$ of $c(\tau)$. This is holomorphic on $\mathcal{F} \times \mathbb{C}$ where $\mathcal{F}$ denotes the fundamental domain of $\Gamma_{2}$, and at least meromorphic on $H_{2} \times \mathbb{C}^{2}$. Now we prove that $F(\tau, z)$ is invariant under $\Gamma_{2}^{J}$. By the properties of $\vartheta_{\nu}$, we see that $\left.F\right|_{1}[(\lambda, \mu)]=F$ for any $\lambda, \mu \in \mathbb{Z}^{2}$. So the problem is the action of $\Gamma_{2}$. Since $\left.\left.F\right|_{k, 1}[\gamma]\right|_{1}[(\lambda, \mu)]=\left.\left.F\right|_{1}\left[(\lambda, \mu) g^{-1}\right]\right|_{k, 1}[\gamma],\left.F\right|_{k, 1}[\gamma]$ also satisfies (4.1). So we have

$$
\left.F\right|_{k, 1}[\gamma]=\sum_{\nu \in(\mathbb{Z} / 2 \mathbb{Z})^{2}} c_{\nu, \gamma}(\tau) \vartheta_{\nu}(\tau, z)
$$

for some holomorphic functions $c_{\nu, \gamma}(\tau)$ on $H_{2}$ which might depend on $\gamma$. For the Taylor expansion

$$
\left.F\right|_{k, 1}[\gamma]=f_{0, \gamma}(\tau)+f_{20, \gamma}(\tau) z_{1}^{2}+f_{11, \gamma}(\tau) z_{1} z_{2}+f_{02, \gamma}(\tau) z_{2}^{2}+\cdots
$$

we put

$$
f_{\gamma}(\tau)=\left(f_{0, \gamma}(\tau), 2 f_{20, \gamma}(\tau) /(2 \pi i)^{2}, f_{11, \gamma}(\tau) /(2 \pi i)^{2}, 2 f_{02, \gamma}(\tau) /(2 \pi i)^{2}\right) .
$$

Then $c_{\gamma}(\tau)=A(\tau)^{-1} f_{\gamma}(\tau)$. As we saw in (3.3) and (3.4), we have

$$
f_{0, \gamma}(\tau)=\xi_{k, 0, \gamma}(\tau)
$$

and

$$
\begin{aligned}
& f_{20, \gamma}(\tau) u^{2}+f_{11, \gamma}(\tau) u_{1} u_{2}+f_{02, \gamma}(\tau) u_{2}^{2} \\
& \quad=\xi_{k, 2, \gamma}(\tau, u)+\frac{2 \pi i}{k}\left(\frac{\partial \xi_{k, 0, \gamma}(\tau)}{\partial \tau_{1}} u_{1}^{2}+\frac{\partial \xi_{k, 0, \gamma}(\tau)}{\partial z_{0}} u_{1} u_{2}+\frac{\partial \xi_{k, 0, \gamma}(\tau)}{\partial \tau_{2}} u_{2}^{2}\right) .
\end{aligned}
$$

Also $\xi_{k, 0, \gamma}(\tau)=\left(\left.\xi_{k, 0}\right|_{k}[\gamma]\right)(\tau)$ and $\xi_{k, 2, \gamma}(\tau, u)=\left(\left.\xi_{k, 2}\right|_{k, 2}[\gamma]\right)(\tau, u)$. But we assumed here that $\left.\xi_{k, 0}\right|_{k}[\gamma]=\xi_{k, 0}$ and $\left.\xi_{k, 2}\right|_{k, 2}[\gamma]=\xi_{k, 2}$, so $f_{\gamma}(\tau)=f(\tau)$. Hence by the uniqueness of solution of $A(\tau) c_{\gamma}(\tau)=f(\tau)$, we have $c_{\nu, \gamma}(\tau)=c_{\nu}(\tau)$ and $\left.F\right|_{k, 1}[\gamma]=F$. 
This means that $F\left(\gamma \tau,{ }^{t}(c \tau+d)^{-1} z\right)=\operatorname{det}(c \tau+d)^{k} e\left({ }^{t}{ }^{t}(c \tau+d)^{-1} z\right) F(\tau, z)$. Since $F(\tau, z)$ is holomorphic on $\mathcal{F} \times \mathbb{C}^{2}$, it is also holomorphic on $\gamma \mathcal{F} \times \mathbb{C}^{2}$, and hence on $H_{2} \times \mathbb{C}^{2}$. So we have proved Theorem 5.1(2).

Now assume that $k$ is odd. In this case the map to $A_{k}\left(\Gamma_{2}\right) \times A_{k, 2}\left(\Gamma_{2}\right)$ is not surjective since $f_{11}(\tau)$ does not necessarily vanish on the diagonals. For odd $k$, any $\xi_{k, 0}(\tau) \in A_{k}\left(\Gamma_{2}\right)$ vanishes at $z_{0}=0$ since they are all multiples of $\chi_{5}(\tau)$ (cf. Igusa [15]). So $f_{11}(\tau)$ vanishes at $z_{0}=0$ if and only if the coefficient of $u_{1} u_{2}$ in $g_{k, 2}(\tau, u)$ vanishes. For such a pair of $g_{k, 0}(\tau)$ and $g_{k, 2}(\tau, u)$ we can define a Jacobi form by the same argument as in the case of even $k$. On the other hand, since the third row of $A(\tau)$ vanishes at $z_{0}=0$, this is also a necessary condition. For odd $k$, the coefficients of $u_{1}^{2}$ and $u_{2}^{2}$ in $g_{k, 2}(\tau, u)$ always vanish by the automorphy with respect to $\operatorname{diag}(1,-1,1,-1)$. So the condition $g_{k, 2}(\tau, u) \in S_{k, 2}^{0}\left(\Gamma_{2}\right)$ is necessary and sufficient for the existence of $F \in J_{k, 1}\left(\Gamma_{2}\right)$ and we have proved (3) of Theorem 5.1.

The generators in Theorem 5.1(4) are given as follows. It is well known from Igusa [15] that $A_{\text {even }}\left(\Gamma_{2}\right)=\mathbb{C}\left[\phi_{4}, \phi_{6}, \chi_{10}, \chi_{12}\right]$, where $\phi_{4}$ and $\phi_{6}$ are the Eisenstein series of weight 4 and 6 respectively and $\chi_{10}$ and $\chi_{12}$ are cusp forms of weight 10 and 12 respectively unique up to a constant. By claim (2) of the theorem, we can take the Jacobi forms $\phi_{4,1}(\tau, z), \phi_{6,1}(\tau, z), \chi_{10,1}(\tau, z)$, and $\chi_{12,1}(\tau, z)$ of weight $k=4,6,10$, and 12 repectively of index 1 such that the image in $A_{k}\left(\Gamma_{2}\right) \times A_{k, 2}\left(\Gamma_{2}\right)$ under $\left(\xi_{k, 0}(\tau), \xi_{k, 2}(\tau, z)\right)$ is $\left(\phi_{4}, 0\right),\left(\phi_{6}, 0\right),\left(\chi_{10}, 0\right)$, and $\left(\chi_{12}, 0\right)$ respectively. Then we can show that the submodule $\widetilde{J}$ of $J_{\text {even,1 }}\left(\Gamma_{2}^{J}\right)$ given by

$$
A_{\text {even }}\left(\Gamma_{2}\right) \phi_{4,1}+A_{\text {even }}\left(\Gamma_{2}\right) \phi_{6,1}+A_{\text {even }}\left(\Gamma_{2}\right) \chi_{10,1}+A_{\text {even }}\left(\Gamma_{2}\right) \chi_{12,1}
$$

is mapped surjectively to $\bigoplus_{k \text { even }}\left(A_{k}\left(\Gamma_{2}\right) \times A_{k, 2}\left(\Gamma_{2}\right)\right)$. This is proved as follows. Firstly, it is obvious that

$$
\bigoplus_{k \text { even }>0} A_{k}\left(\Gamma_{2}\right)=A_{\text {even }}\left(\Gamma_{2}\right) \phi_{4}+A_{\text {even }}\left(\Gamma_{2}\right) \phi_{6}+A_{\text {even }}\left(\Gamma_{2}\right) \chi_{10}+A_{\text {even }}\left(\Gamma_{2}\right) \chi_{12}
$$

(which is not a direct sum). So the mapping of $F(\tau, z) \in \widetilde{J}$ to $F(\tau, 0)$ is surjective onto $\sum_{k>0} A_{k}\left(\Gamma_{2}\right)$. Now we show that the image of $\widetilde{J}$ under $\left(\xi_{k, 0}(\tau), \xi_{k, 2}(\tau, z)\right)$ contains $\{0\} \times A_{k, 2}\left(\Gamma_{2}\right)$. For any $F(\tau) \in A_{k}\left(\Gamma_{2}\right)$ and $G(\tau) \in A_{l}\left(\Gamma_{2}\right)$, we define the Rankin-Cohen type bracket $\{F(\tau), G(\tau)\}_{\mathrm{Sym}(2)}$ as in [19] as follows:

$$
\begin{aligned}
& \{F(\tau), G(\tau)\}_{\operatorname{Sym}(2)}(\tau, u)=\left(l G(\tau) \frac{\partial F(\tau)}{\partial \tau_{1}}-k F(\tau) \frac{\partial G(\tau)}{\partial \tau_{1}}\right) u_{1}^{2} \\
& +\left(l G(\tau) \frac{\partial F(\tau)}{\partial z_{0}}-k F(\tau) \frac{\partial G}{\partial z_{0}}\right) u_{1} u_{2}+\left(l G(\tau) \frac{\partial F(\tau)}{\partial \tau_{2}}-k F(\tau) \frac{\partial G(\tau)}{\partial \tau_{2}}\right) u_{2}^{2}
\end{aligned}
$$

Then $\{F, G\}_{\operatorname{Sym}(2)} \in A_{k+l, 2}\left(\Gamma_{2}\right)$. We take $F(\tau, z) \in J_{k, 1}\left(\Gamma_{2}^{J}\right)$ and $G(\tau, z) \in J_{k, 1}\left(\Gamma_{2}^{J}\right)$ and put $F(\tau)=F(\tau, 0)$ and $G(\tau)=G(\tau, 0)$. We assume that the image of $F$ and $G$ 
in $A_{k}\left(\Gamma_{2}\right) \times A_{k, 2}\left(\Gamma_{2}\right)$ and $A_{l}\left(\Gamma_{2}\right) \times A_{l, 2}\left(\Gamma_{2}\right)$ under $\left(\xi_{k, 0}(\tau), \xi_{k, 2}(\tau, z)\right)$ is $(F(\tau), 0)$ and $(G(\tau), 0)$, respectively. Then the Taylor expansions are given by

$$
\begin{aligned}
& F(\tau, z)=F(\tau)+\frac{2 \pi i}{k}\left(\frac{\partial F(\tau)}{\partial \tau_{1}} u_{1}^{2}+\frac{\partial F(\tau)}{\partial z_{0}} u_{1} u_{2}+\frac{\partial F(\tau)}{\partial \tau_{2}} u_{2}^{2}\right)+O\left(z^{4}\right), \\
& G(\tau, z)=G(\tau)+\frac{2 \pi i}{l}\left(\frac{\partial G(\tau)}{\partial \tau_{1}} u_{1}^{2}+\frac{\partial G(\tau)}{\partial z_{0}} u_{1} u_{2}+\frac{\partial G(\tau)}{\partial \tau_{2}} u_{2}^{2}\right)+O\left(z^{4}\right),
\end{aligned}
$$

where $O\left(z^{4}\right)$ means terms of degree not less than 4 with respect to $z_{1}, z_{2}$. Then

$$
G(\tau) F(\tau, z)-F(\tau) G(\tau, z)=\frac{2 \pi i}{k l}\{F, G\}_{\mathrm{Sym}(2)}(\tau, z)+O\left(z^{4}\right) .
$$

As shown by T. Satoh [19], the $A_{\text {even }}\left(\Gamma_{2}\right)$-module $\bigoplus_{k \text { even }} A_{k, 2}\left(\Gamma_{2}\right)$ is generated by $\left\{\phi_{4}, \phi_{6}\right\}_{\mathrm{Sym}(2)},\left\{\phi_{4}, \chi_{10}\right\}_{\mathrm{Sym}(2)},\left\{\phi_{4}, \chi_{12}\right\}_{\mathrm{Sym}(2)},\left\{\phi_{6}, \phi_{10}\right\}_{\mathrm{Sym}(2)},\left\{\phi_{6}, \phi_{12}\right\}_{\mathrm{Sym}(2)}$, $\left\{\chi_{10}, \chi_{12}\right\}_{\operatorname{Sym}(2)}$. This means that the $A_{\text {even }}\left(\Gamma_{2}\right)$-module spanned by

$$
\begin{array}{ll}
\phi_{4}(\tau) \phi_{6,1}(\tau, z)-\phi_{6}(\tau) \phi_{4,1}(\tau, z), & \phi_{4}(\tau) \chi_{10,1}(\tau, z)-\chi_{10}(\tau) \phi_{4,1}(\tau, z), \\
\phi_{4}(\tau) \chi_{12,1}(\tau, z)-\chi_{12}(\tau) \phi_{4,1}(\tau, z), & \phi_{6}(\tau) \chi_{10,1}(\tau, z)-\chi_{10}(\tau) \phi_{6,1}(\tau, z), \\
\phi_{6}(\tau) \chi_{12,1}(\tau, z)-\chi_{12}(\tau) \phi_{6,1}(\tau, z), & \phi_{10}(\tau) \chi_{12,1}(\tau, z)-\chi_{12}(\tau) \chi_{10,1}(\tau, z),
\end{array}
$$

is mapped surjectively onto

$$
\{0\} \times\left(\bigoplus_{k \text { even }} A_{k, 2}\left(\Gamma_{2}\right)\right) \subset A_{\text {even }}\left(\Gamma_{2}\right) \times\left(\bigoplus_{k \text { even }} A_{k, 2}\left(\Gamma_{2}\right)\right) .
$$

Hence $\widetilde{J}$ is mapped surjectively onto $\sum_{k \text { even }}\left(A_{k}\left(\Gamma_{2}\right) \times A_{k, 2}\left(\Gamma_{2}\right)\right)$. This means that $\widetilde{J}=J_{\text {even, } 2}\left(\Gamma_{2}\right)$ by the injectivity of the mapping; and by the dimension formulas for $A_{k}\left(\Gamma_{2}\right)$ and $A_{k, 2}(\Gamma)$ given below, we see that $\phi_{4,1}, \phi_{6,1}, \chi_{10,1}, \chi_{12,1}$ are free generators over $A_{\text {even }}\left(\Gamma_{2}\right)$. When $k$ is odd, we denote by $\chi_{k, 1}(\tau, z)$ a Jacobi form of index 1 of weight $k=21,27$, or 29 whose image in $A_{k}\left(\Gamma_{2}\right) \times S_{k, 2}^{0}\left(\Gamma_{2}\right)$ is $\left(0, f_{k}\right)$ where $f_{k} \in S_{k, 2}^{0}\left(\Gamma_{2}\right)$ is a non-zero vector valued Siegel modular form. We denote by $\chi_{35,1}$ the Jacobi form of weight 35 of index 1 whose image in $A_{35}\left(\Gamma_{2}\right) \times A_{35,2}\left(\Gamma_{2}\right)$ is $\left(\chi_{35}, 0\right)$. Then obviously $\chi_{21,1}, \chi_{27,1}, \chi_{29,1}, \chi_{35,1}$ are free generators of the $A_{\text {even }}\left(\Gamma_{2}\right)$-module $J_{\text {odd, } 2}\left(\Gamma_{2}\right)$ by the structure theorem in [10] or $[12]$.

We note that the above arguments also give a way to construct generators of Jacobi forms of index one explicitly by giving the coefficients $c_{\nu}(\tau)$ of the theta expansions, since $A_{k}\left(\Gamma_{2}\right), A_{k, 2}\left(\Gamma_{2}\right)$ are known explicitly from [15], [19] and [10], [12]. For the convenience of the readers, we give here the generating functions of related dimensions. The first one is due to Igusa and the others are due to 
Tsushima (cf. [15], [20]-[22]). We have

$$
\begin{aligned}
\sum_{k=0}^{\infty} \operatorname{dim} A_{k}\left(\Gamma_{2}\right) t^{k}= & \frac{1+t^{35}}{\left(1-t^{4}\right)\left(1-t^{6}\right)\left(1-t^{10}\right)\left(1-t^{12}\right)} \\
\sum_{k=0}^{\infty} \operatorname{dim} A_{k, 2}\left(\Gamma_{2}\right) t^{k}= & \frac{t^{10}+t^{14}+2 t^{16}+t^{18}-t^{20}-t^{26}-t^{28}+t^{32}}{\left(1-t^{4}\right)\left(1-t^{6}\right)\left(1-t^{10}\right)\left(1-t^{12}\right)} \\
& +\frac{t^{21}+t^{23}+t^{27}+t^{29}-t^{33}}{\left(1-t^{4}\right)\left(1-t^{6}\right)\left(1-t^{10}\right)\left(1-t^{12}\right)} \\
\sum_{k=1}^{\infty} \operatorname{dim} J_{k, 1}\left(\Gamma_{2}^{J}\right) t^{k}= & \frac{\left(t^{4}+t^{6}+t^{10}+t^{12}\right)+\left(t^{21}+t^{27}+t^{29}+t^{35}\right)}{\left(1-t^{4}\right)\left(1-t^{6}\right)\left(1-t^{10}\right)\left(1-t^{12}\right)}
\end{aligned}
$$

We also have

$$
\sum_{k=0, k \text { odd }}^{\infty} \operatorname{dim} S_{k, 2}^{0}\left(\Gamma_{2}\right) t^{k}=\frac{t^{21}+t^{27}+t^{29}}{\left(1-t^{4}\right)\left(1-t^{6}\right)\left(1-t^{10}\right)\left(1-t^{12}\right)}
$$

This is obtained by an explicit description of $\bigoplus_{k=0, k \text { odd }}^{\infty} S_{k, 2}^{0}\left(\Gamma_{2}\right)$ (cf. [12]).

Since $J_{0,1}\left(\Gamma_{2}^{J}\right)=0$, when we compare the dimensions of the Jacobi forms and Siegel modular forms, we should take the sum only over $k>0$. We have

$$
\begin{aligned}
\sum_{k>0, k \text { even }}^{\infty}\left(\operatorname{dim} A_{k}\left(\Gamma_{2}\right)+\operatorname{dim} A_{k, 2}\left(\Gamma_{2}\right)\right) t^{k} & =\frac{t^{4}+t^{6}+t^{10}+t^{12}}{\left(1-t^{4}\right)\left(1-t^{6}\right)\left(1-t^{10}\right)\left(1-t^{12}\right)}, \\
\sum_{k=1, k \text { odd }}^{\infty}\left(\operatorname{dim} S_{k}\left(\Gamma_{2}\right)+\operatorname{dim} S_{k, 2}^{0}\left(\Gamma_{2}\right)\right) t^{k} & =\frac{t^{21}+t^{27}+t^{29}+t^{35}}{\left(1-t^{4}\right)\left(1-t^{6}\right)\left(1-t^{10}\right)\left(1-t^{12}\right)},
\end{aligned}
$$

and the sum of these gives $\operatorname{dim} J_{k, 1}\left(\Gamma_{2}^{J}\right)$ as in Theorem 5.1.

\section{§6. Explicit structures of index two}

When $n=m=2$, the situation is much more complicated. We assume throughout this section that the weight is even. We put

$$
J_{\text {even }, 2}^{\text {cusp }}\left(\Gamma_{2}^{J}\right)=\bigoplus_{k>0, k \text { even }}^{\infty} J_{k, 2}^{\text {cusp }}\left(\Gamma_{2}^{J}\right) .
$$

In this section we give an explicit structure of $J_{\text {even, } 2}\left(\Gamma_{2}^{J}\right)$ and $J_{\text {even }}^{\text {cusp }}\left(\Gamma_{2}^{J}\right)$ as an $A_{\text {even }}\left(\Gamma_{2}\right)$-module.

Theorem 6.1. The module $J_{\text {even, } 2}\left(\Gamma_{2}^{J}\right)$ is a free $A_{\text {even }}\left(\Gamma_{2}\right)$-module spanned by ten Jacobi forms of respective weights 4, 6, 8, 8, 10, 10, 12, 12, 14, 16. 
As a corollary, the dimension of $J_{k, 2}\left(\Gamma_{2}^{J}\right)$ for even $k$ is given by the generating function

$$
\sum_{k>0, k \text { even }}^{\infty} \operatorname{dim} J_{k, 2}\left(\Gamma_{2}^{J}\right) t^{k}=\frac{t^{4}+t^{6}+2 t^{8}+2 t^{10}+2 t^{12}+t^{14}+t^{16}}{\left(1-t^{4}\right)\left(1-t^{6}\right)\left(1-t^{10}\right)\left(1-t^{12}\right)}
$$

The formula for $\operatorname{dim} J_{k, m}^{\text {cusp }}\left(\Gamma_{2}^{J}\right)$ with $k \geq 4$ or $\operatorname{dim} J_{k, m}\left(\Gamma_{2}^{J}\right)$ with $k \geq 6$ for any $m \geq 0$ has been known to Tsushima, but we need a special care for small $k$, which will be explained later.

Now we give a proof of this theorem based on a comparison between the Taylor expansion and the theta expansion. For any natural number $l$, we denote by $J_{k, 2}^{(l)}\left(\Gamma_{2}^{J}\right)$ the space of Jacobi forms in $J_{k, 2}\left(\Gamma_{2}^{J}\right)$ such that the coefficients of the Taylor expansion vanish up to the total degree $l$ :

$$
J_{k, 2}^{(l)}\left(\Gamma_{2}^{J}\right)=\left\{F \in J_{k, 2}\left(\Gamma_{2}^{J}\right) ;\left.\partial_{1}^{i} \partial_{2}^{j} F\right|_{z_{1}=z_{2}=0}=0 \text { for any } i+j \leq l\right\} .
$$

First of all, we will determine the structure of $J_{k, 2}^{(4)}\left(\Gamma_{2}^{J}\right)$. We start from a concrete description of the theta expansion. When $n=m=2$, there are 16 theta functions $\vartheta_{\nu, 2}(\tau, z)$. But since all the Jacobi forms in $J_{k, m}\left(\Gamma_{2}^{J}\right)$ are even functions of $z$ (i.e. invariant under $z \mapsto-z$ ) when $n=2$, we should take only even functions of $z$ in the basis of theta expansion. So when $m=2$, we put

$$
\begin{aligned}
t_{1}(\tau, z) & =\vartheta_{00,2}(\tau, z), \\
t_{2}(\tau, z) & =\vartheta_{02,2}(\tau, z), \\
t_{3}(\tau, z) & =\vartheta_{20,2}(\tau, z) \\
t_{4}(\tau, z) & =\vartheta_{22,2}(\tau, z) \\
t_{5}(\tau, z) & =\vartheta_{01,2}(\tau, z)+\vartheta_{03,2}(\tau, z), \\
t_{6}(\tau, z) & =\vartheta_{21,2}(\tau, z)+\vartheta_{23,2}(\tau, z), \\
t_{7}(\tau, z) & =\vartheta_{10,2}(\tau, z)+\vartheta_{30,2}(\tau, z), \\
t_{8}(\tau, z) & =\vartheta_{12,2}(\tau, z)+\vartheta_{32,2}(\tau, z), \\
t_{9}(\tau, z) & =\vartheta_{11,2}(\tau, z)+\vartheta_{33,2}(\tau, z)+\vartheta_{13,2}(\tau, z)+\vartheta_{31,2}(\tau, z), \\
t_{10}(\tau, z) & =\vartheta_{11,2}(\tau, z)+\vartheta_{33,2}(\tau, z)-\vartheta_{13,2}(\tau, z)-\vartheta_{31,2}(\tau, z) .
\end{aligned}
$$

Then for all $i$ with $1 \leq i \leq 10$, we have $t_{i}(\tau,-z)=t_{i}(\tau, z)$ and $F(\tau, z) \in J_{k, 2}\left(\Gamma_{2}^{J}\right)$ is a linear combination of these ten theta functions over functions on $H_{2}$. Moreover,

$$
t_{i}\left(\left(\begin{array}{cc}
\tau_{1} & -z_{0} \\
-z_{0} & \tau_{2}
\end{array}\right),\left(\begin{array}{c}
z_{1} \\
-z_{2}
\end{array}\right)\right)=\epsilon_{i} t_{i}(\tau, z)
$$


where $\epsilon_{i}=1$ for $1 \leq i \leq 9$ and -1 for $i=10$. Now for any nine holomorphic functions $F_{i}$ of $H_{2}$ with $1 \leq i \leq 9$, we define $\left\{F_{1}, \ldots, F_{9}\right\}_{13}$ to be the determinant

$$
\left|\begin{array}{cccc}
F_{1} & F_{2} & \cdots & F_{9} \\
\partial_{11} F_{1} & \partial_{11} F_{2} & \cdots & \partial_{11} F_{9} \\
\partial_{12} F_{1} & \partial_{12} F_{2} & \cdots & \partial_{12} F_{9} \\
\partial_{22} F_{1} & \partial_{22} F_{2} & \cdots & \partial_{22} F_{9} \\
\partial_{11}^{2} F_{1} & \partial_{11}^{2} F_{2} & \cdots & \partial_{11}^{2} F_{9} \\
\partial_{11} \partial_{12} F_{1} & \partial_{11} \partial_{12} F_{2} & \cdots & \partial_{11} \partial_{12} F_{9} \\
\left(\partial_{11} \partial_{22}+2 \partial_{12}^{2}\right) F_{1} & \left(\partial_{11} \partial_{22}+2 \partial_{12}^{2}\right) F_{2} & \cdots & \left(\partial_{11} \partial_{22}+2 \partial_{12}^{2}\right) F_{9} \\
\partial_{22} \partial_{12} F_{1} & \partial_{22} \partial_{12} F_{2} & \cdots & \partial_{22} \partial_{12} F_{9} \\
\partial_{22}^{2} F_{1} & \partial_{22}^{2} F_{2} & \cdots & \partial_{22}^{2} F_{9}
\end{array}\right| .
$$

Then

$$
\left\{\left.F_{1}\right|_{1 / 2}[g], \ldots,\left.F_{9}\right|_{1 / 2}[g]\right\}_{13}=\left.\left\{F_{1}, \ldots, F_{9}\right\}_{13}\right|_{9 / 2+13}[g]
$$

for any $g \in S p(2, \mathbb{R})$. This is an easy corollary of Theorem 2 in [9]. For any function $F(\tau, z)$ on $H_{2} \times \mathbb{C}^{2}, g \in S p(2, \mathbb{R})$ and any integer $m \geq 0$, we define

$$
\left.F\right|_{1 / 2, m}[g]=\operatorname{det}(c \tau+d)^{-1 / 2} e^{m}\left(-{ }^{t} z(c \tau+d)^{-1} c z\right) F\left(g \tau,{ }^{t}(c \tau+d)^{-1} z\right) .
$$

Using (4.3), we can show that

$$
\left(\left.t_{1}(\tau, z)\right|_{1 / 2,2}\left[J_{2}\right], \ldots,\left.t_{10}(\tau, z)\right|_{1 / 2,2}\left[J_{2}\right]\right)=\left(t_{1}(\tau, z), \ldots, t_{10}(\tau, z)\right) A_{2}
$$

where

$$
A_{2}=4^{-1} \kappa\left(J_{2}\right)\left(\begin{array}{rrrrrrrrrr}
1 & 1 & 1 & 1 & 2 & 2 & 2 & 2 & 4 & 0 \\
1 & 1 & 1 & 1 & -2 & -2 & 2 & 2 & -4 & 0 \\
1 & 1 & 1 & 1 & 2 & 2 & -2 & -2 & -4 & 0 \\
1 & 1 & 1 & 1 & -2 & -2 & -2 & -2 & 4 & 0 \\
1 & -1 & 1 & -1 & 0 & 0 & 2 & -2 & 0 & 0 \\
1 & -1 & 1 & -1 & 0 & 0 & -2 & 2 & 0 & 0 \\
1 & 1 & -1 & -1 & 2 & -2 & 0 & 0 & 0 & 0 \\
1 & 1 & -1 & -1 & -2 & 2 & 0 & 0 & 0 & 0 \\
1 & -1 & -1 & 1 & 0 & 0 & 0 & 0 & 0 & 0 \\
0 & 0 & 0 & 0 & 0 & 0 & 0 & 0 & 0 & -4
\end{array}\right) .
$$

Since $\kappa\left(J_{2}\right)^{10}=-1$, we have $\operatorname{det}\left(A_{2}\right)=1$. 
We define a $9 \times 10$ matrix $B(\tau)$ of functions on $H_{2}$ by

$$
B(\tau)=\left(\begin{array}{ccc}
t_{1}(\tau, 0) & \cdots & t_{10}(\tau, 0) \\
\left.\partial_{1}^{2} t_{1}(\tau, z)\right|_{z=0} & \cdots & \left.\partial_{1}^{2} t_{10}(\tau, z)\right|_{z=0} \\
\left.\partial_{1} \partial_{2} t_{1}(\tau, z)\right|_{z=0} & \cdots & \left.\partial_{1} \partial_{2} t_{10}(\tau, z)\right|_{z=0} \\
\left.\partial_{2}^{2} t_{1}(\tau, z)\right|_{z=0} & \cdots & \left.\partial_{2}^{2} t_{10}(\tau, z)\right|_{z=0} \\
\left.\partial_{1}^{4} t_{1}(\tau, z)\right|_{z=0} & \cdots & \left.\partial_{1}^{4} t_{10}(\tau, z)\right|_{z=0} \\
\left.\partial_{1}^{3} \partial_{2} t_{1}(\tau, z)\right|_{z=0} & \cdots & \left.\partial_{1}^{3} \partial_{2} t_{10}(\tau, z)\right|_{z=0} \\
\left.\partial_{1}^{2} \partial_{2}^{2} t_{1}(\tau, z)\right|_{z=0} & \cdots & \left.\partial_{1}^{2} \partial_{2}^{2} t_{10}(\tau, z)\right|_{z=0} \\
\left.\partial_{1} \partial_{2}^{3} t_{1}(\tau, z)\right|_{z=0} & \cdots & \left.\partial_{1} \partial_{2}^{3} t(\tau, z)\right|_{z=0} \\
\left.\partial_{2}^{4} t_{1}(\tau, z)\right|_{z=0} & \cdots & \left.\partial_{2}^{4} t_{10}(\tau, z)\right|_{z=0}
\end{array}\right)
$$

By the heat equation, when $a+b$ is even, we have

$$
\partial_{1}^{a} \partial_{2}^{b} t_{i}=8^{(a+b) / 2} \partial_{11}^{(a-c) / 2} \partial_{22}^{(b-c) / 2} \partial_{12}^{c} t_{i}
$$

for any $c \leq \min (a, b)$ with $c \equiv a \equiv b \bmod 2$ and any $1 \leq i \leq 10$. So if we write $t_{i}(\tau)=t_{i}(\tau, 0)$, any $9 \times 9$ minor of $B(\tau)$ excluding the $i$-th column is, up to a common constant, equal to $\widetilde{t}_{i}(\tau):=\left\{t_{1}(\tau), \ldots, t_{i-1}(\tau), t_{i+1}(\tau), \ldots, t_{10}(\tau)\right\}_{13}$. We define a $10 \times 10$ matrix $\widetilde{B}(\tau, z)$ so that the first row is $\left(t_{1}(\tau, z), \ldots, t_{10}(\tau, z)\right)$ and the remaining nine rows are given by $B(\tau)$. We define $F_{18}(\tau, z)$ to be the determinant of $\widetilde{B}(\tau, z)$.

Lemma 6.2. We have $F_{18}(\tau, z) \in J_{k, 2}\left(\Gamma_{2}^{J}\right)$.

Proof. As already explained, we have

$$
F_{18}(\tau, z)=c \sum_{i=1}^{10}(-1)^{i+1} t_{i}(\tau, z) \widetilde{t}_{i}(\tau)
$$

for some non-zero constant $c$, so

$$
\begin{aligned}
\left.F_{18}(\tau, z)\right|_{18,2}\left[J_{2}\right] & =c \sum_{i=1}^{10}(-1)^{i+1}\left(\left.t_{i}(\tau, z)\right|_{1 / 2,2}\left[J_{2}\right]\right) \times\left(\left.\widetilde{t}_{i}(\tau)\right|_{13+9 / 2}\left[J_{2}\right]\right) \\
& =c \sum_{i=1}^{10}(-1)^{i+1}\left(\left.t_{i}(\tau, z)\right|_{1 / 2,2}\left[J_{2}\right]\right) \times \widetilde{t}_{i, J_{2}}(\tau)
\end{aligned}
$$

where we put

$$
\widetilde{t}_{i, J_{2}}(\tau)=\left\{\left.t_{1}(\tau)\right|_{1 / 2}\left[J_{2}\right], \ldots,\left.t_{i-1}(\tau)\right|_{1 / 2}\left[J_{2}\right],\left.t_{i+1}(\tau)\right|_{1 / 2}\left[J_{2}\right], \ldots,\left.t_{10}(\tau)\right|_{1 / 2}\left[J_{2}\right]\right\}_{13} .
$$


Hence by taking $\partial_{1}^{i} \partial_{2}^{j}$ of both sides of (6.1) for various $i$ and $j$ and restricting to $z=0$, we see that

$$
\left.F_{18}(\tau, z)\right|_{18,2}\left[J_{2}\right]=\operatorname{det}\left(A_{2}\right) F_{18}(\tau, z)=F_{18}(\tau, z)
$$

As for $t(U)$ with $U \in G L_{2}(\mathbb{Z})$, the action gives a permutation on $\vartheta_{\nu, 2}$ up to sign and using the same argument as above, we can show that $F_{18}(\tau, z)$ is invariant under this action. In the same way we can show that $u(S)$ acts trivially.

Since the $10 \times 10$ matrix defining $F_{18}(\tau, z)$ contains $z$ only in the first row, $\left.\partial_{1}^{i} \partial_{2}^{j} F_{18}(\tau, z)\right|_{z=0}$ for any $i, j$ with $i+j \leq 4$ is the determinant of a matrix whose first row is zero or which has two identical rows. So it vanishes, i.e.

$$
\left.\partial_{1}^{i} \partial_{2}^{j} F(\tau, z)\right|_{z=0}=0
$$

for all $i+j \leq 4$. In other words, the Taylor coefficients of $F_{18,2}(\tau, z)$ vanish up to degree 4 .

Theorem 6.3. (1) $F_{18}(\tau, z)$ is not identically zero and belongs to $J_{18,2}^{(4)}\left(\Gamma_{2}^{J}\right)$.

(2) $F_{18}(\tau, z)$ is divisible by $\chi_{10}(\tau)=\chi_{5}(\tau)^{2} \in S_{10}\left(\Gamma_{2}\right)$.

(3) If we put $F_{8}(\tau, z)=F_{18}(\tau, z) / \chi_{10}(\tau)$, then $F_{8}(\tau, z) \in J_{8,2}^{\text {cusp }}\left(\Gamma_{2}^{J}\right)$.

(4) When $k$ is even, we have $J_{k, 2}^{(4)}\left(\Gamma_{2}^{J}\right)=F_{8}(\tau, z) A_{k-8}\left(\Gamma_{2}\right)$. All such Jacobi forms are Jacobi cusp forms. In particular, $J_{8,2}^{(4)}\left(\Gamma_{2}^{J}\right)=\mathbb{C} F_{8}(\tau, z)$ and $J_{k, 2}^{(4)}\left(\Gamma_{2}^{J}\right)=0$ for $k<8$.

Proof. To prove non-vanishing of $F_{18}(\tau, z)$, it is sufficient to show that the coefficient of $t_{10}(\tau, z)$, which is a non-zero constant multiple of $\left\{t_{1}(\tau), \ldots, t_{9}(\tau)\right\}_{13}$, does not vanish, and this is not difficult to show. But here we shall show instead that the Taylor coefficient of $F_{18}(\tau, z)$ at $z_{1}^{3} z_{2}^{3}$ does not vanish, since we need this later. We define $\partial_{i}$ and $\partial_{i j}$ as before. By the heat equation we see that for any fixed $a, b$ with $a+b$ even and $1 \leq l \leq 10$, the functions $\partial_{11}^{(a-c) / 2} \partial_{22}^{(b-c) / 2} \partial_{12}^{c} t_{l}(\tau)$ are the same for any non-negative integer $c$ with $c \leq \min (a, b)$ such that $a \equiv b \equiv c \bmod 2$. For $l=1, \ldots, 9$, we write

$$
t_{l}(\tau)=t_{l}(\tau, 0)=f_{l}\left(\tau_{1}, \tau_{2}\right)+z_{0}^{2} g_{l}\left(\tau_{1}, \tau_{2}\right)+O\left(z_{0}^{4}\right),
$$

where $\tau=\left(\begin{array}{cc}\tau_{1} & z_{0} \\ z_{0} & \tau_{2}\end{array}\right)$. Since $\partial_{12}^{2} t_{l}=\partial_{11} \partial_{22} t_{l}$, we have

$$
g_{l}\left(\tau_{1}, \tau_{2}\right)=\frac{(4 \pi i)^{2}}{2} \partial_{11} \partial_{22} f_{l}\left(\tau_{1}, \tau_{2}\right),
$$


SO

$$
\begin{aligned}
& \partial_{12} t_{l}(\tau)=(4 \pi i) z_{0} \partial_{11} \partial_{22} f_{l}\left(\tau_{1}, \tau_{2}\right)+O\left(z_{0}^{3}\right), \\
& \partial_{12}^{2} t_{l}(\tau)=\partial_{11} \partial_{22} f_{l}\left(\tau_{1}, \tau_{2}\right)+O\left(z_{0}^{2}\right) .
\end{aligned}
$$

We also have

$$
\partial_{12} t_{10}(\tau)=\frac{1}{2} \eta\left(\tau_{1}\right)^{3} \eta\left(\tau_{2}\right)^{3}+O\left(z_{0}^{2}\right)
$$

where $\eta(\tau)$ is the Dedekind eta function. This is because

$$
\eta(\tau)^{3}=\sum_{p \in \mathbb{Z}}\left(\left(2 p+\frac{1}{2}\right) e\left(2\left(p+\frac{1}{4}\right)^{2} \tau\right)-\left(2 p+\frac{3}{2}\right) e\left(2\left(p+\frac{3}{4}\right)^{2} \tau\right)\right)
$$

by the classical results. We put $t(\tau)=\left(t_{1}(\tau), \ldots, t_{10}(\tau)\right)$ and $v=v\left(\tau_{1}, \tau_{2}\right)=$ $\left(f_{1}\left(\tau_{1}, \tau_{2}\right), \ldots, f_{9}\left(\tau_{1}, \tau_{2}\right)\right)$. Now the Taylor coefficient of $F_{18}(\tau, z)$ at $z_{1}^{3} z_{2}^{3}$ is up to a non-zero constant equal to $\left.\partial_{1}^{3} \partial_{2}^{3} \operatorname{det}(\widetilde{B}(\tau, z))\right|_{z=0}$, i.e. the determinant of the $10 \times 10$ matrix given by $\left(\begin{array}{c}\partial_{1}^{3} \partial_{2}^{3} t(\tau) \\ B(\tau)\end{array}\right)$. By the relations we gave above, we have

$$
\begin{gathered}
\left(\begin{array}{c}
\partial_{11} \partial_{22} \partial_{12} t(\tau) \\
t(\tau) \\
\partial_{11} t(\tau) \\
\partial_{12} t(\tau)-(4 \pi i) z_{0} \partial_{12}^{2} t(\tau) \\
\partial_{22} t(\tau) \\
\partial_{11}^{2} t(\tau) \\
\partial_{11} \partial_{12} t(\tau) \\
\partial_{11} \partial_{22} t(\tau) \\
\partial_{22} \partial_{12} t(\tau) \quad \\
\partial_{22}^{2} t(\tau) \quad
\end{array}\right) \\
\left(\begin{array}{cc}
(4 \pi i) z_{0} \partial_{11}^{2} \partial_{22}^{2} v & \\
v+O\left(z_{0}^{2}\right) & \\
\partial_{11} v+O\left(z_{0}^{2}\right) & \\
O\left(z_{0}^{3}\right) & \\
\partial_{22} v+O\left(z_{0}^{2}\right) & (2 \pi i) z_{0} \eta\left(\tau_{1}\right)^{3} \eta\left(\tau_{2}\right)^{3}+O\left(z_{0}^{3}\right) \\
\partial_{11}^{2} v+O\left(z_{0}^{2}\right) & (2 \pi i) z_{0} \eta\left(\tau_{1}\right)^{3} \eta\left(\tau_{2}\right)^{3}+O\left(z_{0}^{3}\right) \\
(4 \pi i) z_{0} \partial_{11}^{2} \partial_{22} v+O\left(\tau_{2}\right)^{3}+O\left(z_{0}^{3}\right) & \\
\partial_{11} \partial_{22} v+O\left(z_{0}^{2}\right) & (2 \pi i) z_{0} \eta\left(\tau_{1}\right)^{3} \eta\left(\tau_{2}\right)^{3}+O\left(z_{0}^{3}\right) \\
(4 \pi i) z_{0} \partial_{11} \partial_{22}^{2} v+O\left(z_{0}^{3}\right) & \partial_{11} \partial_{22}^{2} \eta\left(\tau_{1}\right)^{3} \eta\left(\tau_{2}\right)^{3} / 2 \\
\partial_{22}^{2} v+O\left(z_{0}^{2}\right) & (2 \pi i) z_{0} \eta\left(\tau_{1}\right)^{3} \eta\left(\tau_{2}\right)^{3}+O\left(z_{0}^{3}\right)
\end{array}\right) .
\end{gathered}
$$

We expand the determinant of this matrix along the last column. We see that the 1st, 7 -th and 9 -th rows are divisible by $z_{0}$ except for the 10 -th component, and the 
4-th row is divisible by $z_{0}^{3}$ except for the last component. From this we see that the cofactor at $(i, 10)$ for $i \neq 4$ is $O\left(z_{0}^{4}\right)$, so calculating the $(4,10)$ cofactor which is apparently $O\left(z_{0}^{3}\right)$, we see that the determinant of the above matrix is equal to

$$
2^{-1}(4 \pi i)^{3} \eta\left(\tau_{1}\right)^{3} \eta\left(\tau_{2}\right)^{3} z_{0}^{3} \times \operatorname{det}\left(\begin{array}{c}
\partial_{11}^{2} \partial_{22}^{2} v \\
\partial_{11} v \\
\partial_{22} v \\
\partial_{11}^{2} v \\
\partial_{11}^{2} \partial_{22} v \\
\partial_{11} \partial_{22} v \\
\partial_{11} \partial_{22}^{2} v \\
\partial_{22}^{2} v
\end{array}\right)+O\left(z_{0}^{4}\right)
$$

We show that this is not zero by showing that the determinant part of the above expression, which is independent of $z_{0}$, is non-zero. We have

$$
\begin{aligned}
& \left.t_{1}(\tau, 0)\right|_{z_{0}=0}=\vartheta_{0,2}\left(\tau_{1}\right) \vartheta_{0,2}\left(\tau_{2}\right), \\
& \left.t_{2}(\tau, 0)\right|_{z_{0}=0}=\vartheta_{0,2}\left(\tau_{1}\right) \vartheta_{2,2}\left(\tau_{2}\right), \\
& \left.t_{3}(\tau, 0)\right|_{z_{0}=0}=\vartheta_{2,2}\left(\tau_{1}\right) \vartheta_{0,2}\left(\tau_{2}\right), \\
& \left.t_{4}(\tau, 0)\right|_{z_{0}=0}=\vartheta_{2,2}\left(\tau_{1}\right) \vartheta_{2,2}\left(\tau_{2}\right) \\
& \left.t_{5}(\tau, 0)\right|_{z_{0}=0}=\vartheta_{0,2}\left(\tau_{1}\right)\left(\vartheta_{1,2}\left(\tau_{2}\right)+\vartheta_{3,2}\left(\tau_{2}\right)\right), \\
& \left.t_{6}(\tau, 0)\right|_{z_{0}=0}=\vartheta_{2,2}\left(\tau_{1}\right)\left(\vartheta_{1,2}\left(\tau_{2}\right)+\vartheta_{1,3}\left(\tau_{2}\right)\right), \\
& \left.t_{7}(\tau, 0)\right|_{z_{0}=0}=\left(\vartheta_{1,2}\left(\tau_{1}\right)+\vartheta_{3,2}\left(\tau_{1}\right) \vartheta_{0,2}\left(\tau_{1}\right),\right. \\
& \left.t_{8}(\tau, 0)\right|_{z_{0}=0}=\left(\vartheta_{1,2}\left(\tau_{1}\right)+\vartheta_{3,2}\left(\tau_{1}\right)\right) \vartheta_{2,2}\left(\tau_{1}\right), \\
& \left.t_{9}(\tau, 0)\right|_{z_{0}=0}=\left(\vartheta_{1,2}\left(\tau_{1}\right)+\vartheta_{3,2}\left(\tau_{1}\right)\right)\left(\vartheta_{1,2}\left(\tau_{2}\right)+\vartheta_{3,2}\left(\tau_{2}\right)\right) .
\end{aligned}
$$

So if we put

$$
C\left(\tau_{i}\right)=\left(\begin{array}{ccc}
\vartheta_{0,2}\left(\tau_{i}\right) & \vartheta_{2,2}\left(\tau_{i}\right) & \vartheta_{1,2}\left(\tau_{i}\right)+\vartheta_{3,2}\left(\tau_{i}\right) \\
\partial_{i i} \vartheta_{0,2}\left(\tau_{i}\right) & \partial_{i i} \vartheta_{2,2}\left(\tau_{i}\right) & \partial_{i i}\left(\vartheta_{1,2}\left(\tau_{i}\right)+\vartheta_{3,2}\left(\tau_{i}\right)\right) \\
\partial_{i i}^{2} \vartheta_{0,2}\left(\tau_{i}\right) & \partial_{i i}^{2} \vartheta_{2,2}\left(\tau_{i}\right) & \partial_{i i}^{2}\left(\vartheta_{1,2}\left(\tau_{i}\right)+\vartheta_{3,2}\left(\tau_{i}\right)\right)
\end{array}\right),
$$

then we easily see that, up to a non-zero constant, $\left.\partial_{1}^{3} \partial_{2}^{3} F_{18}(\tau, z)\right|_{z=0}$ is equal to $\eta\left(\tau_{1}\right)^{3} \eta\left(\tau_{2}\right)^{3} \times z_{0}^{3} \operatorname{det}\left(C\left(\tau_{1}\right) \otimes C\left(\tau_{2}\right)\right)+O\left(z_{0}^{4}\right)$. Since $\operatorname{det}\left(C\left(\tau_{i}\right)\right)$ is equal to $\eta\left(\tau_{i}\right)^{15}$ up to a non-zero constant (cf. e.g. Kramer [17]), the function $\left.\partial_{1}^{3} \partial_{2}^{3} F_{18}(\tau, z)\right|_{z=0}$ is equal to $z_{0}^{3} \Delta\left(\tau_{1}\right)^{2} \Delta\left(\tau_{2}\right)^{2}+O\left(z_{0}^{4}\right)$ up to a non-zero constant. So this is not zero and we see that the coefficient of $\left(z_{1} z_{2}\right)^{3}$ in $F_{18}(\tau, z)$ is non-zero.

To show that $F_{18}(\tau, z)$ is divisible by $\chi_{10}$, we show that the coefficients $c_{18, l}(\tau)$ $(1 \leq l \leq 10)$ of the theta expansion of $F_{18}(\tau, z)$ are divisible by $\chi_{10}$. By definition of $F_{18}(\tau, z)$, each $c_{18, l}(\tau)$ is a $9 \times 9$ minor of $B(\tau)$. Expanding minors of $B(\tau)$ at $z_{0}=0$ 
in the same way as above, we see that $c_{18,10}(\tau)$ is divisible by $z_{0}^{5}$ and $c_{18, l}(\tau)$ with $1 \leq l \leq 9$ is divisible by $z_{0}^{2}$. So $c_{18, l}(\tau) / \chi_{10}(\tau)$ and $F_{18}(\tau, z)$ are holomorphic for any $\tau$ in the fundamental domain of $\Gamma_{2}$. Since $F_{18}(\tau, z) \in J_{k, 2}\left(\Gamma_{2}^{J}\right), F_{18}(\tau, z) / \chi_{10}(\tau)$ is holomorphic on the whole $H_{2} \times \mathbb{C}^{2}$.

If we denote by $c_{8, l}(\tau)$ the coefficient in $t_{l}(\tau, z)$ of the theta expansion of $F_{8}(\tau, z)$, then the vector $c_{8}(\tau)=\left(c_{8, l}(\tau)\right)_{1 \leq l \leq 10}$ is a solution of the simultaneous equation $B(\tau) c_{8}(\tau)=0$. Since the first $9 \times 9$ minor of $B(\tau)$ is $z_{0} \eta\left(\tau_{1}\right)^{15} \eta\left(\tau_{2}\right)^{15}$ up to a non-zero constant, the rank of $B(\tau)$ is 9 as a matrix of meromorphic functions. Hence any such solution is $\left(a(\tau) c_{8, l}(\tau)\right)_{1 \leq l \leq 10}$ for some meromorphic function $a(\tau)$ on $H_{2}$. So if $F(\tau, z) \in J_{k, 2}^{(4)}\left(\Gamma_{2}^{J}\right)$, then $F(\tau, z)=a(\tau) F_{8}(\tau, z)$ for some meromorphic function $a(\tau)$. Now we must show that $a(\tau)$ is holomorphic. This is not trivial at all, since if we take $F_{18}(\tau, z)$ instead for example, then even if we multiply by $1 / \chi_{10}$ which is not holomorphic, we get a holomorphic Jacobi form $F_{8}(\tau, z)$. Now denote by $f_{8,3}(\tau, z)$ the term of total degree six of the Taylor expansion of $F_{8}(\tau, z)$. It is non-zero. This obviously comes from the corresponding fact for $F_{18}(\tau, z)$ that we have shown above. Since the Taylor coefficients of total degree $0,1,2$ of $F_{8}(\tau, z)$ are zero for the same reason, we have $f_{8,3}(\tau, z) \in A_{8,6}\left(\Gamma_{2}\right)$. Now we need the following theorem.

Theorem 6.4 ([11], [12]). The module $\bigoplus_{k \text { even }} A_{k, 6}\left(\Gamma_{2}\right)$ is a free $A_{\text {even }}\left(\Gamma_{2}\right)$-module spanned by seven Siegel modular forms in $A_{6,6}\left(\Gamma_{2}\right), A_{8,6}\left(\Gamma_{2}\right), A_{10,6}\left(\Gamma_{2}\right)$, $A_{12,6}\left(\Gamma_{2}\right), A_{14,6}\left(\Gamma_{2}\right), A_{16,6}\left(\Gamma_{2}\right)$, or $A_{18,6}\left(\Gamma_{2}\right)$.

Since $\operatorname{dim} A_{8,6}\left(\Gamma_{2}\right)=1$, Theorem 6.4 means that $f_{8,3}(\tau, u)$ is one of the free generators of $\bigoplus_{k \text { even }} A_{k, 6}\left(\Gamma_{2}\right)$. Denote by $g(\tau, z)$ the degree 6 part of the Taylor expansion of $F(\tau, z)=a(\tau) F_{8}(\tau, z) \in J_{k, 2}^{(4)}\left(\Gamma_{2}^{J}\right)$. Then $g(\tau, u)=a(\tau) f_{8,3}(\tau, u)$ and $f_{8,3}(\tau, u)$ is one of the free generators in Theorem 6.4 , so $a(\tau)$ must be holomorphic. This means that $a(\tau) \in A_{k-8}\left(\Gamma_{2}\right)$ and $J_{k, 2}^{(4)}\left(\Gamma_{2}^{J}\right)=F_{8}(\tau, z) A_{k-8}\left(\Gamma_{2}\right)$.

Now assume that $0 \neq F(\tau, z) \in J_{8,2}^{\text {cusp }}\left(\Gamma_{2}^{J}\right)$. By the dimension formulas of Igusa [15] and Tsushima [20]-[22], we know that $\operatorname{dim} J_{8,2}^{\text {cusp }}\left(\Gamma_{2}^{J}\right)=1, \operatorname{dim} S_{8}\left(\Gamma_{2}\right)=$ $\operatorname{dim} A_{8,2}\left(\Gamma_{2}\right)=\operatorname{dim} S_{8,4}\left(\Gamma_{2}\right)=0$. Since the image of the mapping from $J_{k, 2}^{\text {cusp }}\left(\Gamma_{2}^{J}\right)$ to $A_{k}\left(\Gamma_{2}\right) \times A_{k, 2}\left(\Gamma_{2}\right) \times A_{k, 4}\left(\Gamma_{2}\right)$ must be cusp forms, the Taylor coefficients of degree $0,2,4$ of $F(\tau, z)$ should be zero. So $F \in J_{k, 2}^{(4)}\left(\Gamma_{2}^{J}\right)$ and hence $F$ is a constant multiple of $F_{8}$. So $F_{8}$ is a Jacobi cusp form. Thus we have proved Theorem 6.3.

Now we prove Theorem 6.1. This is based on the following theorem.

Theorem 6.5. (1) For any even $k>0$, the mapping from $J_{k, 2}^{\text {cusp }}\left(\Gamma_{2}^{J}\right)$ to $S_{k}\left(\Gamma_{2}\right) \times$ $S_{k, 2}\left(\Gamma_{2}\right) \times S_{k, 4}\left(\Gamma_{2}\right)$ induced by Theorem 3.1 is surjective.

(2) For any even $k>0$, the mapping from $J_{k, 2}\left(\Gamma_{2}^{J}\right)$ to $A_{k}\left(\Gamma_{2}\right) \times A_{k, 2}\left(\Gamma_{2}\right) \times A_{k, 4}\left(\Gamma_{2}\right)$ is surjective. 
Proof. In both (1) and (2), the kernel of the mapping is $F_{8}(\tau, z) A_{k-8}\left(\Gamma_{2}\right)$. We know all the dimensions in (1), so (1) is reduced to a comparison of dimensions found by Igusa and Tsushima. Indeed we have

$$
\begin{aligned}
& \sum_{k>0, k \text { even }} \operatorname{dim} A_{k}\left(\Gamma_{2}\right) t^{k}=\frac{1}{\left(1-t^{4}\right)\left(1-t^{6}\right)\left(1-t^{10}\right)\left(1-t^{12}\right)}-1 \\
& =\frac{t^{4}+t^{6}+t^{12}-t^{14}-2 t^{16}-t^{18}+t^{20}+t^{26}+t^{28}-t^{32}}{\left(1-t^{4}\right)\left(1-t^{6}\right)\left(1-t^{10}\right)\left(1-t^{12}\right)}, \\
& \sum_{k \text { even }} \operatorname{dim} S_{k, 2}\left(\Gamma_{2}\right) t^{k}=\frac{t^{14}+2 t^{16}+t^{18}+t^{22}-t^{26}-t^{28}}{\left(1-t^{4}\right)\left(1-t^{6}\right)\left(1-t^{10}\right)\left(1-t^{12}\right)}, \\
& \sum_{k \text { even }} \operatorname{dim} S_{k, 4}\left(\Gamma_{2}\right) t^{k}=\frac{t^{10}+t^{12}+t^{14}+t^{16}+t^{18}+t^{20}-t^{30}}{\left(1-t^{4}\right)\left(1-t^{6}\right)\left(1-t^{10}\right)\left(1-t^{12}\right)} \\
& \sum_{k \text { even } \geq 8} \operatorname{dim} A_{k-8}\left(\Gamma_{2}\right) t^{k}=\frac{t^{8}}{\left(1-t^{4}\right)\left(1-t^{6}\right)\left(1-t^{10}\right)\left(1-t^{12}\right)}, \\
& \sum_{k \text { even }>0} \operatorname{dim} J_{k, 2}^{\text {cusp }}\left(\Gamma_{2}^{J}\right) t^{k} \\
& =\frac{t^{8}+2 t^{10}+2 t^{12}+2 t^{14}+3 t^{16}+2 t^{18}+t^{20}-t^{26}-t^{28}-t^{30}}{\left(1-t^{4}\right)\left(1-t^{6}\right)\left(1-t^{10}\right)\left(1-t^{12}\right)}
\end{aligned}
$$

so for even $k>0$, we have

$$
\operatorname{dim} J_{k, 2}^{\text {cusp }}\left(\Gamma_{2}\right)=\operatorname{dim} S_{k}\left(\Gamma_{2}\right)+\operatorname{dim} S_{k, 2}\left(\Gamma_{2}\right)+\operatorname{dim} S_{k, 4}\left(\Gamma_{2}\right)+\operatorname{dim} A_{k-8}\left(\Gamma_{2}\right) .
$$

Since $\operatorname{dim} J_{k, 2}^{(4)}\left(\Gamma_{2}\right)=A_{k-8}\left(\Gamma_{2}\right)$, this means the surjectivity of (1). Next we prove (2). By [4], for $k \geq 6$ we have $\operatorname{dim} J_{k, 2}\left(\Gamma_{2}\right)=\operatorname{dim} J_{k, 2}^{\text {cusp }}\left(\Gamma_{2}^{J}\right)+\operatorname{dim} J_{k, 2}\left(\Gamma_{1}^{J}\right)$ and

$$
\begin{aligned}
\sum_{k=1}^{\infty} \operatorname{dim} J_{k, 2}\left(\Gamma_{1}^{J}\right) t^{k} & =\frac{t^{4}+t^{6}+t^{8}+t^{11}}{\left(1-t^{4}\right)\left(1-t^{6}\right)} \\
\sum_{k \text { even }} \operatorname{dim} A_{k, 4}\left(\Gamma_{2}\right) t^{k} & =\frac{t^{8}+t^{10}+t^{12}+t^{14}+t^{16}}{\left(1-t^{4}\right)\left(1-t^{6}\right)\left(1-t^{10}\right)\left(1-t^{12}\right)}
\end{aligned}
$$

So we can calculate $\operatorname{dim} J_{k, 2}\left(\Gamma_{2}^{J}\right)$ and for even $k \geq 6$ we can show that

$$
\operatorname{dim} J_{k, 2}\left(\Gamma_{2}\right)=\operatorname{dim} A_{k}\left(\Gamma_{2}\right)+\operatorname{dim} A_{k, 2}\left(\Gamma_{2}\right)+\operatorname{dim} A_{k, 4}\left(\Gamma_{2}\right)+\operatorname{dim} A_{k-8}\left(\Gamma_{2}\right) .
$$

Since the kernel of the mapping from $J_{k, 2}\left(\Gamma_{2}^{J}\right)$ to $A_{k}\left(\Gamma_{2}\right) \times A_{k, 2}\left(\Gamma_{2}\right) \times A_{k, 4}\left(\Gamma_{2}\right)$ is again $J_{k, 2}^{(4)}\left(\Gamma_{2}\right)$, the above relation means the surjectivity for $k \geq 6$ again in this case. When $k=2$ or $k=4$, since $J_{k, 2}^{(4)}\left(\Gamma_{2}^{J}\right)=0$, the above mapping is injective. Since we know that $A_{2, j}\left(\Gamma_{2}\right)=0$ for $j=0,2,4$ and $\operatorname{dim} A_{4}\left(\Gamma_{2}\right)=1, A_{4,2}\left(\Gamma_{2}\right)=$ 
$A_{4,4}\left(\Gamma_{2}\right)=0$, we have $\operatorname{dim} J_{2,2}\left(\Gamma_{2}^{J}\right)=0$ and $\operatorname{dim} J_{4,2}\left(\Gamma_{2}^{J}\right) \leq 1$. If we take the theta function $E_{4}^{(3)}$ of degree 3 associated with the unique even unimodular lattice of rank 8, then $E_{4}^{(3)}\left(\begin{array}{cc}\tau & 0 \\ 0 & \tau_{33}\end{array}\right)=\phi_{4}(\tau) e_{4}\left(\tau_{33}\right)$ where $\tau \in H_{2}, \tau_{33} \in H_{1}$, and $e_{4}\left(\tau_{33}\right)$ is the Eisenstein series of weight $k$ of degree one. Take the second Fourier-Jacobi coefficient $F_{4}(\tau, z)$ of $E_{4}^{(3)}$ with respect to $\tau_{33}$. Then since $e_{4}\left(\tau_{33}\right)=1+240\left(e\left(\tau_{33}\right)+\right.$ $\left.9 e\left(2 \tau_{33}\right)+\cdots\right)$, we have $F_{4}(\tau, 0)=240 \cdot 9 \phi_{4}(\tau)$ and this gives a non-zero Jacobi form in $J_{4,2}\left(\Gamma_{2}^{J}\right)$. So we have proved claim (2) of Theorem 6.5.

In order to prove Theorem 6.1, we give generators of $J_{\text {even, } 2}\left(\Gamma_{2}^{J}\right)$ as an $A_{\text {even }}\left(\Gamma_{2}\right)$-module. In the explanation below, we also give an alternative proof of claim (2) of Theorem 6.5, assuming (1) but without using the dimension formula for $J_{k, 2}\left(\Gamma_{2}^{J}\right)$, since this will make the situation clearer. First we show that the mapping from $F(\tau, z) \in J_{k, 2}\left(\Gamma_{2}^{J}\right)$ to $\xi_{k, 0}(\tau)=F(\tau, 0) \in A_{k}\left(\Gamma_{2}\right)$ is surjective. It is enough to give Jacobi forms $F(\tau, z)$ of index 2 such that $F(\tau, 0)$ is $\phi_{4}, \phi_{6}, \chi_{10}$, or $\chi_{12}$. For $k=10,12$, there exist Jacobi cusp forms $\chi_{k, 2}(\tau, z) \in J_{k, 2}\left(\Gamma_{2}^{J}\right)$ such that $\chi_{10,2}(\tau, 0)=\chi_{10}(\tau)$ and $\chi_{12,2}(\tau, 0)=\chi_{12}(\tau)$ by claim $(1)$. For later use, we take $\chi_{k, 2}(\tau, 2)$ so that the image in $A_{k}\left(\Gamma_{2}\right) \times A_{k, 2}\left(\Gamma_{2}\right) \times A_{k, 4}\left(\Gamma_{2}\right)$ is $\left(\chi_{k, 2}(\tau, 0), 0,0\right)$ for $k=10,12$. We can do so by claim (1). The case of $\phi_{4}$ has already been explained above. When $k=6$, we take the Eisenstein series $E_{6}^{(3)}$ of degree 3 of weight 6 and $F_{6,2}(\tau, z)$ can be defined by the second Fourier-Jacobi coefficient of $E_{6}^{(3)}$. The non-vanishing of $F_{6,2}$ can be proved in the same way and we have $F_{6}(\tau, 0)=-540 \cdot 33 \phi_{6}(\tau)$. We write $\phi_{4,2}=F_{4,2} /(240 \cdot 9)$ and $\phi_{6,2}=F_{6,2} /(-540 \cdot 33)$, so $\phi_{k, 2}(\tau, 0)=\phi_{k}$ for $k=4,6$. So we have proved the mapping from $J_{k, 2}\left(\Gamma_{2}\right)$ to $A_{k}\left(\Gamma_{2}\right)$ is surjective. Since $A_{k, 2}\left(\Gamma_{2}\right)=A_{k, 4}\left(\Gamma_{2}\right)=\{0\}$ for $k=2,4$, the image of $\phi_{k, 2}(\tau, z)$ in $A_{k}\left(\Gamma_{2}\right) \times A_{k, 2}\left(\Gamma_{2}\right) \times A_{k, 4}\left(\Gamma_{2}\right)$ for $k=4$ or 6 is $\left(\phi_{k}, 0,0\right)$. Now we write

$$
\widetilde{J}_{2}=A_{\text {even }}\left(\Gamma_{2}\right) \phi_{4,2}(\tau, z)+A_{\text {even }}\left(\Gamma_{2}\right) \phi_{6,2}(\tau, z)+A_{\text {even }}\left(\Gamma_{2}\right) \chi_{10,2}+A_{\text {even }}\left(\Gamma_{2}\right) \chi_{12,2}
$$

Next we show that $\widetilde{J}_{2}$ is surjectively mapped onto $A_{k}\left(\Gamma_{2}\right) \times A_{k, 2}\left(\Gamma_{2}\right)$ by $\left(\xi_{k, 0}(\tau), \xi_{k, 2}(\tau, z)\right)$. Indeed we can prove this in almost the same way as in the proof of Theorem 5.1(4). For $F(\tau, z) \in J_{k, 2}\left(\Gamma_{2}^{J}\right)$, write $F(\tau)=F(\tau, 0)$ and assume that $\xi_{k, 2}(\tau, u)=0$ for this $F$. Then we have

$$
F(\tau, z)=F(\tau)+\frac{4 \pi i}{k}\left(\frac{\partial F(\tau)}{\partial \tau_{1}} z_{1}^{2}+\frac{\partial F(\tau)}{\partial z_{0}} z_{1} z_{2}+\frac{\partial F(\tau)}{\partial \tau_{2}} z_{2}^{2}\right)+\cdots
$$

We take another $G(\tau, z) \in J_{l, 2}\left(\Gamma_{2}^{J}\right)$ such that the image in $A_{k}\left(\Gamma_{2}\right) \times A_{k, 2}\left(\Gamma_{2}\right)$ is $(G(\tau), 0)$, where we put $G(\tau)=G(\tau, 0)$. If we define the Rankin-Cohen type bracket $\{F(\tau), G(\tau)\}_{\text {Sym (2) }}$ as before, we can show as in the proof of Theorem 5.1 
that the $A_{\text {even }}\left(\Gamma_{2}\right)$-module spanned by

$$
\begin{array}{ll}
\phi_{4}(\tau) \phi_{6,2}(\tau, z)-\phi_{6}(\tau) \phi_{4,2}(\tau, z), & \phi_{4}(\tau) \chi_{10,2}(\tau, z)-\chi_{10}(\tau) \phi_{4,2}(\tau, z), \\
\phi_{4}(\tau) \chi_{12,2}(\tau, z)-\chi_{12}(\tau) \phi_{4,2}(\tau, z), & \phi_{6}(\tau) \chi_{10,2}(\tau, z)-\chi_{10}(\tau) \phi_{6,2}(\tau, z), \\
\phi_{6}(\tau) \chi_{12,2}(\tau, z)-\chi_{12}(\tau) \phi_{6,2}(\tau, z), & \phi_{10}(\tau) \chi_{12,2}(\tau, z)-\chi_{12}(\tau) \chi_{10,2}(\tau, z),
\end{array}
$$

is mapped surjectively onto

$$
\{0\} \times\left(\bigoplus_{k \text { even }} A_{k, 2}\left(\Gamma_{2}\right)\right) \subset A_{\text {even }}\left(\Gamma_{2}\right) \times\left(\bigoplus_{k \text { even }} A_{k, 2}\left(\Gamma_{2}\right)\right) .
$$

Finally we show that the map is surjective onto $\{0\} \times\{0\} \times A_{k, 4}\left(\Gamma_{2}\right) \subset A_{k}\left(\Gamma_{2}\right) \times$ $A_{k, 2}\left(\Gamma_{2}\right) \times A_{k, 4}\left(\Gamma_{2}\right)$. By Theorem $6.5(1)$, we already know that the image contains $S_{k, 4}\left(\Gamma_{2}\right)$. We have $\operatorname{dim} A_{8,4}\left(\Gamma_{2}\right)=1$ and $\operatorname{dim} S_{8,4}\left(\Gamma_{2}\right)=0$. So we first construct a non-zero Jacobi form $G_{8}(\tau, z) \in J_{8,2}\left(\Gamma_{2}^{J}\right)$ whose image in $A_{8}\left(\Gamma_{2}\right) \times A_{8,2}\left(\Gamma_{2}\right)$ is zero and non-zero in $A_{8,4}\left(\Gamma_{2}\right)$. By Theorem 5.1, we have a unique Jacobi form $\phi_{4,1} \in$ $J_{4,1}\left(\Gamma_{2}^{J}\right)$ such that $\phi_{4,1}(\tau, 0)=\phi_{4}$. We put $G_{8}(\tau, z)=\phi_{4}(\tau) \phi_{4,2}(\tau, z)-\phi_{4,1}(\tau, z)^{2}$. Then obviously $G_{8}(\tau, z) \in J_{8,2}\left(\Gamma_{2}^{J}\right)$. Since $G_{8}(\tau, 0)=\phi_{4}(\tau)^{2}-\phi_{4}(\tau)^{2}=0$ and $A_{8,2}\left(\Gamma_{2}\right)=0$, the Taylor coefficients of $G_{8}(\tau, z)$ up to degree 2 are zero. We consider the Taylor coefficient of degree 4. Since $A_{4,2}\left(\Gamma_{2}\right)=A_{4,4}\left(\Gamma_{2}\right)=0$, we have $\xi_{4,2}(\tau, u)=\xi_{4,4}(\tau, u)=0$ for $\phi_{4,1}(\tau, z)$ and $\phi_{4,2}(\tau, z)$, and the Taylor coefficients of these forms are determined by $\phi_{4}$ up to degree 4 as follows:

$$
\begin{aligned}
& \phi_{4,1}(\tau, z)=\phi_{4}(\tau)+\frac{2 \pi i}{4} \mathcal{D}_{2}\left(\phi_{4}\right)(\tau, z)+\frac{(2 \pi i)^{2}}{40}\left(\mathcal{D}_{2}^{2} \phi_{4}\right)(\tau, z)+\cdots, \\
& \phi_{4,2}(\tau, z)=\phi_{4}(\tau)+\frac{4 \pi i}{4}\left(\mathcal{D}_{2} \phi_{4}\right)(\tau, z)+\frac{(2 \pi i)^{2}}{10}\left(\mathcal{D}_{2}^{2} \phi_{4}\right)(\tau, z)+\cdots .
\end{aligned}
$$

Here $\mathcal{D}_{2}$ is defined as in (3.1). So we have

$$
\begin{aligned}
& \phi_{4}(\tau) \phi_{4,2}(\tau, z)-\phi_{4,1}(\tau, z)^{2} \\
& \quad=\frac{(2 \pi i)^{2}}{80}\left(\left.4 \phi_{4}(\tau)\left(\mathcal{D}_{2}^{2} \phi_{4}\right)(\tau, u)\right|_{u=z}-5\left(\left.\left(\mathcal{D}_{2} \phi_{4}\right)(\tau, u)\right|_{u=z}\right)^{2}\right)+O\left(z^{6}\right)
\end{aligned}
$$

We can show that $4 \phi_{4} \mathcal{D}_{2}^{2} \phi-4\left(\mathcal{D}_{2} \phi_{4}\right)^{2} \neq 0$ in many ways. For example if we apply the $\Phi$-operator to the coefficient of $z_{1}^{4}$, then up to a constant we obtain $4 e_{4}\left(\tau_{1}\right) e_{4}^{\prime \prime}\left(\tau_{1}\right)-5\left(e_{4}^{\prime}(\tau)\right)^{2}=\Delta\left(\tau_{1}\right)$, which is non-zero. Hence the image of $G_{8}(\tau, z)$ in $A_{8,4}\left(\Gamma_{2}\right)$ is non-zero. Now we use the following theorem of [11], [12].

Theorem 6.6. The module $\bigoplus_{k \text { even }} A_{k, 4}\left(\Gamma_{2}\right)$ is a free $A_{\text {even }}\left(\Gamma_{2}\right)$-module spanned by certain five forms belonging respectively to $A_{8,4}\left(\Gamma_{2}\right), S_{10,4}\left(\Gamma_{2}\right), S_{12,4}\left(\Gamma_{2}\right)$, $S_{14,4}\left(\Gamma_{2}\right)$ and $S_{16,4}\left(\Gamma_{2}\right)$. 
By Theorem 6.5(1), for each $k=10,12,14,16$, there exists a Jacobi cusp form $\chi_{k, 2 b}(\tau, z)$ whose image in $A_{k}\left(\Gamma_{2}\right) \times A_{k, 2}\left(\Gamma_{2}\right) \times A_{k, 4}\left(\Gamma_{2}\right)$ is $\left(0,0, F_{k}\right)$ where $F_{k}$ is the generator in $S_{k, 4}\left(\Gamma_{2}\right)$ of Theorem 6.6. If we take linear combinations over $A_{\text {even }}\left(\Gamma_{2}\right)$ of these forms together with $G_{8}(\tau, z)$, for each $k$ we obtain the space of Jacobi forms whose image in $A_{k}\left(\Gamma_{2}\right) \times A_{k, 2}\left(\Gamma_{2}\right) \times A_{k, 4}\left(\Gamma_{2}\right)$ is equal to $\{0\} \times\{0\} \times A_{k, 4}\left(\Gamma_{2}\right)$. So we have proved that for any even $k>0$, the mapping from $J_{k, 2}\left(\Gamma_{2}\right)$ to $A_{k}\left(\Gamma_{2}\right) \times A_{k, 2}\left(\Gamma_{2}\right) \times A_{k, 4}\left(\Gamma_{2}\right)$ is surjective.

Proof of Theorem 6.1. Now we see that the Jacobi forms $F_{8,2}(\tau, z), \phi_{4,2}, \phi_{6,2}$, $\chi_{10,2}, \chi_{12,2}, G_{8,2}(\tau, z), \chi_{10,2 b}, \chi_{12,2 b}, \chi_{14,2 b}, \chi_{16,2 b}$ are free generators of $J_{\text {even }, 2}\left(\Gamma_{2}^{J}\right)$ over $A_{\text {even }}\left(\Gamma_{2}\right)$. Indeed if we denote by $J$ the module of Jacobi forms generated by these ten Jacobi forms over $A_{\text {even }}\left(\Gamma_{2}\right)$, then by the above argument, we see that $J$ is mapped surjectively onto $A_{k}\left(\Gamma_{2}\right) \times A_{k, 2}\left(\Gamma_{2}\right) \times A_{k, 4}\left(\Gamma_{2}\right)$ by $\left(\xi_{k, 0}(\tau), \xi_{k, 2}(\tau, z), \xi_{k, 4}(\tau, z)\right)$. We have already proved that the kernel of this mapping is $F_{8,2}(\tau, z) A_{k-8}\left(\Gamma_{2}\right)$ in Theorem 6.3. Therefore in order that the dimension $\operatorname{dim}\left(J \cap J_{k, 2}\left(\Gamma_{2}^{J}\right)\right)$ attains the value $\operatorname{dim} A_{k}\left(\Gamma_{2}\right)+\operatorname{dim} A_{k, 2}\left(\Gamma_{2}\right)+\operatorname{dim} A_{k, 4}\left(\Gamma_{2}\right)+$ $\operatorname{dim} A_{k-8}\left(\Gamma_{2}\right)$ for every even $k$, these ten Jacobi forms should be free over $A_{\text {even }}\left(\Gamma_{2}\right)$. So we have proved Theorem 6.1 completely.

Remark 6.7. We give a remark on higher indices. For index 3 and even $k$, we have the following relations between dimensions:

$$
\begin{aligned}
& \operatorname{dim} J_{k, 3}^{\mathrm{cusp}}\left(\Gamma_{2}^{J}\right)=\operatorname{dim} S_{k}\left(\Gamma_{2}\right)+\operatorname{dim} S_{k, 2}\left(\Gamma_{2}\right)+\operatorname{dim} S_{k, 4}\left(\Gamma_{2}\right)+\operatorname{dim} S_{k, 6}\left(\Gamma_{2}\right) \\
& \quad+\operatorname{dim} A_{k-12}\left(\Gamma_{2}\right)+\operatorname{dim} A_{k-14}\left(\Gamma_{2}\right)+\operatorname{dim} A_{k-18}\left(\Gamma_{2}\right)+\operatorname{dim} A_{k-20}\left(\Gamma_{2}\right), \\
& \operatorname{dim} J_{k, 3}\left(\Gamma_{2}^{J}\right)=\operatorname{dim} A_{k}\left(\Gamma_{2}\right)+\operatorname{dim} A_{k, 2}\left(\Gamma_{2}\right)+\operatorname{dim} A_{k, 4}\left(\Gamma_{2}\right)+\operatorname{dim} A_{k, 6}\left(\Gamma_{2}\right) \\
& \quad+\operatorname{dim} A_{k-12}\left(\Gamma_{2}\right)+\operatorname{dim} A_{k-14}\left(\Gamma_{2}\right)+\operatorname{dim} A_{k-18}\left(\Gamma_{2}\right)+\operatorname{dim} A_{k-20}\left(\Gamma_{2}\right) .
\end{aligned}
$$

So it is plausible that $\bigoplus_{k \text { even }} J_{k, 3}^{(6)}\left(\Gamma_{2}^{J}\right)$ is a free module generated by four Jacobi cusp forms of weights $12,14,18,20$ over $A_{\text {even }}\left(\Gamma_{2}\right)$ and the map from $J_{k, 3}\left(\Gamma_{2}^{J}\right)$ to $A_{k}\left(\Gamma_{2}\right) \times A_{k, 2}\left(\Gamma_{2}\right) \times A_{k, 4}\left(\Gamma_{2}\right) \times A_{k, 6}\left(\Gamma_{2}\right)$ given by $\left(\xi_{k, 0}, \xi_{k, 2}, \xi_{k, 4}, \xi_{k, 6}\right)$ is surjective. When the index is higher than 3 , it seems that the situation is more complicated.

\section{$\S 7$. Image of the Witt operator}

For any function $G(\tau)$ of $H_{2}$, we often use the restriction of $G$ to the diagonals defined by

$$
W G\left(\tau_{1}, \tau_{2}\right)=G\left(\begin{array}{cc}
\tau_{1} & 0 \\
0 & \tau_{2}
\end{array}\right)
$$


This operator $W$ is usually called the Witt operator. In the same way, for any $F(\tau, z) \in J_{k, 1}\left(\Gamma_{2}^{J}\right)$, we define a holomorphic function on $\left(H_{1} \times \mathbb{C}\right)^{2}$ by

$$
(W F)\left(\tau_{1}, z_{1}, \tau_{2}, z_{2}\right)=F\left(\left(\begin{array}{cc}
\tau_{1} & 0 \\
0 & \tau_{2}
\end{array}\right),\left(\begin{array}{c}
z_{1} \\
z_{2}
\end{array}\right)\right) .
$$

By abuse of language, we call this operator on Jacobi forms also the Witt operator. By the automorphy of $F$ with respect to the elements

$$
\left(\begin{array}{cccc}
a_{1} & 0 & b_{1} & 0 \\
0 & a_{2} & 0 & b_{2} \\
c_{1} & 0 & d_{1} & 0 \\
0 & c_{2} & 0 & d_{2}
\end{array}\right) \in \Gamma_{2}
$$

where $a_{i} d_{i}-c_{i} d_{i}=1$ for $i=1,2$ and the Heisenberg part $H(\mathbb{Z})$, we see that $W F$ is a Jacobi form with respect to each variable $\left(\tau_{1}, z_{1}\right)$ or $\left(\tau_{2}, z_{2}\right)$ for each fixed $\left(\tau_{2}, z_{2}\right)$ or $\left(\tau_{1}, z_{1}\right)$. If $k$ is odd, then it is known that $J_{k, 1}\left(\Gamma_{1}^{J}\right)=0$, so we have $W\left(J_{k, 1}\left(\Gamma_{2}^{J}\right)\right)=0$. When $k$ is even, by the action of

$$
\left(\begin{array}{llll}
0 & 1 & 0 & 0 \\
1 & 0 & 0 & 0 \\
0 & 0 & 0 & 1 \\
0 & 0 & 1 & 0
\end{array}\right) \in \Gamma_{2}
$$

on $F(\tau, z) \in J_{k, 1}\left(\Gamma_{2}^{J}\right)$, we see that $(W F)\left(\tau_{1}, z_{1}, \tau_{2}, z_{2}\right)$ is invariant under the exchange of variables $\left(\tau_{1}, z_{1}\right)$ and $\left(\tau_{2}, z_{2}\right)$. It follows that $W F$ is in the symmetric tensors $\operatorname{Sym}^{2}\left(J_{k, 1}\left(\Gamma_{1}^{J}\right)\right)$ of the second degree, i.e.

$$
W F=\sum_{i, j}\left(f_{i}\left(\tau_{1}, z_{1}\right) g_{j}\left(\tau_{2}, z_{2}\right)+g_{j}\left(\tau_{1}, z_{1}\right) f_{i}\left(\tau_{2}, z_{2}\right)\right)
$$

for some $f_{i}, g_{j} \in J_{k, 1}\left(\Gamma_{1}^{J}\right)$. B. Heim asked the author if this map is surjective. We show that the answer is affirmative below.

Theorem 7.1. The Witt operator on $J_{k, 1}\left(\Gamma_{2}^{J}\right)$ is surjective onto $\operatorname{Sym}^{2}\left(J_{k, 1}\left(\Gamma_{1}^{J}\right)\right)$.

Proof. When $k$ is odd, this is trivial since the target of the mapping is zero. So we assume that $k$ is even. The Witt operator is surjective if $\operatorname{dim} \operatorname{ker} W \mid J_{k, 1}\left(\Gamma_{2}^{J}\right)=$ $\operatorname{dim} J_{k, 1}\left(\Gamma_{2}^{J}\right)-\operatorname{dim} \operatorname{Sym}^{2}\left(J_{k, 1}\left(\Gamma_{1}^{J}\right)\right)$. For even $k>0$ by [5] we have

$$
\operatorname{dim} J_{k, 1}\left(\Gamma_{1}^{J}\right)=\operatorname{dim} A_{k}\left(\Gamma_{1}\right)+\operatorname{dim} S_{k+2}\left(\Gamma_{1}\right)= \begin{cases}k / 6 & \text { if } k \equiv 0 \bmod 6 \\ (k-2) / 6 & \text { if } k \equiv 2 \bmod 6 \\ (k+2) / 6 & \text { if } k \equiv 4 \bmod 6\end{cases}
$$


So we have

$$
\begin{aligned}
\sum_{k \text { even }} & \operatorname{dim} \operatorname{Sym}^{2}\left(J_{k, 1}\left(\Gamma_{1}^{J}\right)\right) t^{k} \\
& =\frac{1}{2}\left(\sum_{m=1}^{\infty} m(m+1) t^{6 m}+\sum_{m=0}^{\infty} m(m+1) t^{6 m+2}+\sum_{m=0}^{\infty}(m+1)(m+2) t^{6 m+4}\right) \\
& =\frac{t^{6}}{\left(1-t^{6}\right)^{3}}+\frac{t^{8}}{\left(1-t^{6}\right)^{3}}+\frac{t^{10}}{\left(1-t^{6}\right)^{3}}+\frac{t^{4}}{\left(1-t^{6}\right)^{2}} \\
& =\frac{t^{4}}{\left(1-t^{2}\right)\left(1-t^{6}\right)^{2}}=\frac{t^{4}+t^{6}+t^{10}+t^{12}}{\left(1-t^{4}\right)\left(1-t^{6}\right)\left(1-t^{12}\right)},
\end{aligned}
$$

so by using the formula for $\operatorname{dim} J_{k, 1}\left(\Gamma_{2}^{J}\right)$, it is enough to show that

$$
\sum_{k=1, k \text { even }}^{\infty} \operatorname{dim}\left(\operatorname{ker}\left(W \mid J_{k, 1}\left(\Gamma_{2}^{J}\right)\right)\right) t^{k}=\frac{t^{10}\left(t^{4}+t^{6}+t^{10}+t^{12}\right)}{\left(1-t^{4}\right)\left(1-t^{6}\right)\left(1-t^{10}\right)\left(1-t^{12}\right)}
$$

Now assume that $F(\tau, z) \in J_{k, 1}\left(\Gamma_{2}^{J}\right)$ for even $k$ and $W F=0$. We have $W \vartheta_{\nu_{1} \nu_{2}}(\tau, z)$ $=\vartheta_{\nu_{1}}\left(\tau_{1}, z_{1}\right) \vartheta_{\nu_{2}}\left(\tau_{2}, z_{2}\right)$, and the four functions $\vartheta_{l}\left(\tau_{i}, z_{i}\right)(l=0,1, i=1,2)$ are algebraically independent. So writing $F(\tau, z)=\sum_{\nu \in(\mathbb{Z} / 2 \mathbb{Z})^{2}} c_{\nu}(\tau) \vartheta_{\nu}(\tau, z)$, the condition $W F=0$ is equivalent to $W c_{\nu}=0$ for all $\nu \in(\mathbb{Z} / 2 \mathbb{Z})^{2}$. We write $\tau=\left(\begin{array}{ll}\tau_{1} & z_{0} \\ z_{0} & \tau_{2}\end{array}\right) \in H_{2}$. First we assume that $W F=0$. Then the images of the Taylor coefficients under $W$ at $z=0$ also vanish. By the automorphy with respect to $\operatorname{diag}(1,-1,1,-1) \in \Gamma_{2}$ for even $k$, we have

$$
F\left(\left(\begin{array}{cc}
\tau_{1} & -z_{0} \\
-z_{0} & \tau_{2}
\end{array}\right),\left(\begin{array}{c}
z_{1} \\
-z_{2}
\end{array}\right)\right)=F\left(\left(\begin{array}{cc}
\tau_{1} & z_{0} \\
z_{0} & \tau_{2}
\end{array}\right),\left(\begin{array}{c}
z_{1} \\
z_{2}
\end{array}\right)\right)
$$

This means that the Taylor coefficients of $F(\tau, z)$ at $1, z_{1}^{2}, z_{2}^{2}$ are even functions of $z_{0}^{2}$. But $W c_{\nu}=0$ by the assumption that $W F=0$, so the order is at least one, so the Taylor coefficients at $1, z_{1}^{2}, z_{2}^{2}$ vanish at least of order two at $z_{0}=0$. As for the coefficient of $z_{1} z_{2}$ of $F(\tau, z)$, we can prove the same thing as follows. By definition, we see that

$$
\left.\left(\left.\partial_{1} \partial_{2} \vartheta_{\nu}(\tau, z)\right|_{z=0}\right)\right|_{z_{0}=0}=\prod_{i=1}^{2} \sum_{p_{i} \in \mathbb{Z}}\left(p_{i}+\nu_{i} / 2\right) e\left(\left(p+\nu_{i} / 2\right)^{2} \tau_{i}\right)=0 .
$$

So the Taylor coefficients of $\vartheta_{\nu}(\tau, z)$ at $z_{1} z_{2}$ vanish at $z_{0}=0$. Since $c_{\nu}$ also vanishes at $z_{0}=0$, the vanishing order at $z_{0}=0$ of the Taylor coefficient of $F(\tau, z)$ at $z_{1} z_{2}$ is also at least two. So we have proved that if $W F=0$ then each Taylor coefficient up to degree two vanishes at $z_{0}=0$ of order two. Conversely, take any $F \in J_{k, 1}\left(\Gamma_{2}^{J}\right)$ and assume that each Taylor coefficient at $1, z_{1}^{2}, z_{1} z_{2}, z_{2}^{2}$ vanishes at least of 
order two at $z_{0}=0$. As shown in Section 4 , the coefficients $c_{\nu}(\tau)$ of the theta expansion of $F$ form a solution of the simultaneous equation $A(\tau) c(\tau)=f(\tau)$ where $\operatorname{det} A(\tau)=\chi_{5}(\tau)$ whose vanishing order at $z_{0}=0$ is one and $f(\tau)$ consists of the Taylor coefficients at $1, z_{1}^{2}, z_{1} z_{2}, z_{2}^{2}$. So from $c(\tau)=A(\tau)^{-1} f(\tau)$, we see that $W c_{\nu}(\tau)=0$, and hence $W F=0$. So the condition $W F=0$ is equivalent to the condition that its Taylor coefficients vanish at $z_{0}=0$ of order two up to degree two. By Theorem 5.1, we have a linear isomorphism of $J_{k, 1}\left(\Gamma_{2}^{J}\right)$ onto $A_{k}\left(\Gamma_{2}\right) \times A_{k, 2}\left(\Gamma_{2}\right)$, and this mapping is constructed by using the Taylor coefficients up to degree two, so in order to calculate $\operatorname{dim} \operatorname{ker}(W)$, we interpret the above condition as a condition on the image in $A_{k}\left(\Gamma_{2}\right) \times A_{k, 2}\left(\Gamma_{2}\right)$. Writing the Taylor expansion $F(\tau, z)=f_{0}(\tau)+f_{2}(\tau, z)+\cdots$ as the sum of homogeneous parts $f_{k}(\tau, z)$ of degree $k$, and defining $\xi_{k, 0}(\tau) \in A_{k}\left(\Gamma_{2}\right)$ and $\xi_{k, 2}(\tau, u) \in A_{k, 2}\left(\Gamma_{2}\right)$ for $F$ as in Section 2, we have

$$
\begin{aligned}
\xi_{k, 0}(\tau) & =f_{0}(\tau) \\
\xi_{k, 2}(\tau, u) & =f_{2}(\tau, u)+\frac{2 \pi i}{k}\left(\frac{\partial f_{0}(\tau)}{\partial \tau_{1}} u_{1}^{2}+\frac{\partial f_{0}(\tau)}{\partial z_{0}} u_{1} u_{2}+\frac{\partial f_{0}(\tau)}{\partial \tau_{2}} u_{2}^{2}\right)
\end{aligned}
$$

Here we demand that $f_{0}(\tau)$ and $f_{2}(\tau, u)$ have zero at $z_{0}=0$ at least of order two. It is well known that for $f_{0} \in A_{k}\left(\Gamma_{2}\right)$ with even $k$, the condition $W f_{0}=0$ is equivalent to $f_{0}$ being divisible by $\chi_{10}$. So write $f_{0}(\tau)=\chi_{10}(\tau) f(\tau)$ with $f \in A_{k-10}\left(\Gamma_{2}\right)$. In particular, if $k<10$, then $f_{0}=f=0$. Since $A_{k, 2}\left(\Gamma_{2}\right)=0$ if $k<10$ (cf. [19]), we also have $\xi_{k, 2}(\tau, z)=0$, so $f_{2}(\tau, z)=0$. This means that the corresponding Jacobi form $F$ is 0 . We examine derivatives of $f_{0}=f \chi_{10}$. The form $\chi_{10}$ vanishes at $z_{0}=0$ exactly of order two. So the derivatives of $\chi_{10}$ with respect to $\tau_{i}$ for $i=1,2$ have also zero at $z_{0}=0$ of order two. As for the derivative with respect to $z_{0}$, we have

$$
\frac{\partial f_{0}}{\partial z_{0}}=\frac{\partial \chi_{10}}{\partial z_{0}} f+\chi_{10} \frac{\partial f}{\partial z_{0}}
$$

This vanishes at $z_{0}=0$ at least of order one. So $W \xi_{k, 2}(\tau, z)=0$. This means that $\xi_{k, 2}(\tau, z)$ is divisible by $\chi_{5}(\tau)$. Since $\chi_{5}(\tau)$ is a cusp form of $\Gamma_{2}$ with multiplier, $\xi_{k, 2}(\tau, z)$ is also a cusp form. We have $\operatorname{dim} A_{10,2}\left(\Gamma_{2}\right)=1$ but $S_{10,2}\left(\Gamma_{2}\right)=0$. So $\xi_{10,2}(\tau, z)=0$. But if $k=10$, then $f$ is a constant. If moreover $f \neq 0$, then $\frac{\partial f_{0}}{\partial z_{0}}$ has zero at $z_{0}=0$ exactly of order one. This contradicts the assumption that $\xi_{10,2}(\tau, z)=0$ and $f_{2}(\tau, z)$ is divisible by $z_{0}^{2}$. So we have $f=0$ and $F=0$ also in this case. Hence we can assume now that $k>10$. The function $\frac{\partial f_{0}}{\partial z_{0}}$ has zero at least of order two at $z_{0}=0$ if and only if $W f=0$. So if $W f=0$, then since $f_{2}(\tau, z)$ and the first derivatives of $f_{0}(\tau)$ have zero at least of order two at $z_{0}=0$, so does $\xi_{k, 2}(\tau, z)$ and we have $\xi_{k, 2}(\tau, z) \in \chi_{10} A_{k, 2}\left(\Gamma_{2}\right)$ in this case. So the image of $\operatorname{ker}\left(W \mid J_{k, 1}\left(\Gamma_{2}^{J}\right)\right)$ in $A_{k}\left(\Gamma_{2}\right) \times A_{k, 2}\left(\Gamma_{2}\right)$ contains the space of 
$\left(\chi_{10} f, \xi_{k, 2}(\tau, z)\right)$ with $W f=0$ and $W \xi_{k, 2}=0$. Now we consider the part $W f \neq 0$. We have $\left\{f, \chi_{10}\right\}_{\operatorname{Sym}(2)} \in A_{k, 2}\left(\Gamma_{2}\right)$ and the coefficient of $u_{1} u_{2}$ in this form is $10 \chi_{10} \frac{\partial f}{\partial z_{0}}-(k-10) f \frac{\partial \chi_{10}}{\partial z_{0}}$. Since the function

$$
\frac{2 \pi i}{k(k-10)}\left(10 \chi_{10} \frac{\partial f}{\partial z_{0}}-(k-10) f \frac{\partial \chi_{10}}{\partial z_{0}}\right)+\frac{2 \pi i}{k} \frac{\partial f_{0}}{\partial z_{0}}=\left(10+\frac{2 \pi i}{k}\right) \chi_{10} \frac{\partial f}{\partial z_{0}}
$$

has zero at least of order two at $z_{0}=0$ and the coefficient of $u_{i}^{2}$ of $\left\{f, \chi_{10}\right\}_{\operatorname{Sym}(2)}$ always has zero at least of order two at $z_{0}=0$ for each $i=1,2$, we see that

$$
\xi_{k, 2}(\tau, z)+\frac{2 \pi i}{k(k-10)}\left\{\chi_{10}, f\right\}_{\mathrm{Sym}(2)}
$$

has zero of order two at $z_{0}=0$ and belongs to $\chi_{10} A_{k-10,2}\left(\Gamma_{2}\right)$. If $W f=0$, then $\chi_{10}, f \in \chi_{10} A_{k-10}\left(\Gamma_{2}\right)$, so including the case $W f=0$, we have

$$
\begin{aligned}
\xi_{k, 0}(\tau) & =\chi_{10} f, \\
\xi_{k, 2}(\tau, u) & \in-\frac{2 \pi i}{k(k-10)}\left\{\chi_{10}, f\right\}_{\operatorname{Sym}(2)}+\chi_{10} A_{k-10,2}\left(\Gamma_{2}\right)
\end{aligned}
$$

for some $f \in A_{k-10}\left(\Gamma_{2}\right)$. Conversely, if $\xi_{k, 0}(\tau)$ and $\xi_{k, 2}(\tau, u)$ are written like this, then obviously the Taylor coefficients of $F$ vanish at least of order two at $z_{0}=0$ up to degree two terms, so $F$ belongs to the kernel of $W$. So for $k>10$,

$$
\operatorname{dim} \operatorname{ker}\left(W \mid J_{k, 1}\left(\Gamma_{2}^{J}\right)\right)=\operatorname{dim} A_{k-10}\left(\Gamma_{2}\right)+\operatorname{dim} A_{k-10,2}\left(\Gamma_{2}\right) .
$$

Since the dimension is 0 for $k \leq 10$, the generating function of $\operatorname{dim} \operatorname{ker}\left(W \mid J_{k, 1}\left(\Gamma_{2}^{J}\right)\right)$ is given by

$$
\sum_{k=2, \text { even }}^{\infty}\left(\operatorname{dim} A_{k-10}\left(\Gamma_{2}\right)+\operatorname{dim} A_{k-10,2}\left(\Gamma_{2}\right)\right) t^{k}=\frac{\left(t^{4}+t^{6}+t^{10}+t^{12}\right) t^{10}}{\left(1-t^{4}\right)\left(1-t^{6}\right)\left(1-t^{10}\right)\left(1-t^{12}\right)}
$$

as desired. So we have proved Theorem 7.1.

We note that even if all the Taylor coefficients at $1, z_{1}^{2}, z_{1} z_{2}, z_{2}^{2}$ of $F \in J_{k, 1}\left(\Gamma_{2}^{J}\right)$ vanish under $W$, this does not mean that $W F=0$. Conceptually this is explained by the fact that $\operatorname{Sym}^{2}\left(J_{k, 1}\left(\Gamma_{1}^{J}\right)\right)$ is determined by the coefficients of $1, z_{1}^{2}, z_{2}^{2}, z_{1}^{2} z_{2}^{2}$, and the Taylor coefficients of $W F$ up to degree two are not enough to determine this. For example, if we define $\chi_{10,1}$ as in the proof of Theorem 5.1 by the Jacobi form in $J_{10,1}\left(\Gamma_{2}^{J}\right)$ which is mapped to $\left(\chi_{10}, 0\right) \in A_{10}\left(\Gamma_{2}\right) \times A_{10,2}\left(\Gamma_{2}\right)$, then this is a counterexample. Indeed the Taylor coefficients of $W \chi_{10,1}$ at $1, z_{1}^{2}, z_{2}^{2}$ vanish but the coefficient of $z_{1}^{2} z_{2}^{2}$ is a non-zero constant multiple of $\Delta\left(\tau_{1}\right) \Delta\left(\tau_{2}\right)$. This follows by the above argument or also by giving $c_{\nu}(\tau)$ explicitly by solving the equation 
$A(\tau) c(\tau)=f(\tau)$ where $f(\tau)$ is defined for $\xi_{10,0}=\chi_{10}$ and $\xi_{10,2}=0$ as in $\S 5$. By the result of [12], we see that

$$
\begin{aligned}
\sum_{k=2, \text { even }}^{\infty}\left(\operatorname{dim} \operatorname{ker}\left(W \mid A_{k}\left(\Gamma_{2}\right)\right)+\operatorname{dim} \operatorname{ker}\left(W \mid A_{k, 2}\left(\Gamma_{2}\right)\right)\right) t^{k} & \\
= & \frac{t^{10}+t^{14}+t^{16}+t^{22}}{\left(1-t^{4}\right)\left(1-t^{6}\right)\left(1-t^{10}\right)\left(1-t^{12}\right)}
\end{aligned}
$$

This does not coincide with the generating function of $\operatorname{dim} \operatorname{ker}\left(W \mid J_{k, 2}\left(\Gamma_{2}^{J}\right)\right)$.

It would be interesting to consider the same question for higher degree cases.

\section{Acknowledgements}

This research was partly supported by Grant-in-Aid for Scientific Research (No. 21244001), Japan Society for the Promotion of Science.

The author would like to thank Samuel Grushevsky for a discussion in June in 2009 at Osaka, which definitely convinced the author that the vanishing order of the Taylor coefficients of Jacobi forms of higher degree is much more complicated than the case of degree one.

\section{References}

[1] T. Arakawa, Jacobi Eisenstein series and a basis problem for Jacobi forms, Comment. Math. Univ. St. Pauli 43 (1994), 181-216. Zbl 0837.11027 MR 1302305

[2] H. Aoki and T. Ibukiyama, Simple graded rings of Siegel modular forms, differential operators and Borcherds products, Int. J. Math. 16 (2005), 249-279. Zbl 1068.11030 MR 2130626

[3] S. Böcherer, Über die Fourier-Jacobi-Entwicklung Siegelscher Eisensteinreihen, Math. Z. 183 (1983), 21-46, (II) ibid. 189 (1985), 81-110. Zbl 0558.10022 MR 0776540

[4] J. Dulinski, A decomposition theorem for Jacobi forms, Math. Ann. 303 (1995), 473-498. Zbl 0882.11027 MR 1355001

[5] M. Eichler and D. Zagier, The theory of Jacobi forms, Progr. Math. 55, Birkhäuser Boston, Boston, MA, 1985. Zbl 0554.10018 MR 0781735

[6] E. Freitag, Siegelsche Modulfunktionen, Grundlehren Math. Wiss. 254, Springer, 1983. Zbl 0498.10016 MR 0871067

[7] S. Hayashida and T. Ibukiyama, Siegel modular forms of half integral weight and a lifting conjecture, J. Math. Kyoto Univ. 45 (2005), 489-530. Zbl 1122.11028 MR 2206360

[8] T. Ibukiyama, On Jacobi forms and Siegel modular forms of half integral weights, Comment. Math. Univ. St. Pauli 41 (1992), 109-124. Zbl 0787.11015 MR 1190152

[9] - On differential operators on automorphic forms and invariant pluri-harmonic polynomials, Comment. Math. Univ. St. Pauli 48 (1999), 103-118. Zbl 1007.11023 MR 1684769

[10] Differential operators and structures of vector valued Siegel modular forms, in Algebraic number theory and related topics, Sūriken Kōkyūroku 1200, RIMS, Kyoto Univ., 2001, 71-81 (in Japanese). Zbl 0985.11503 MR 1854835 
[11] Vector valued Siegel modular forms of $\operatorname{Sym}(4)$ and $\operatorname{Sym}(6)$, in Study of automorphic forms and their Dirichlet series, Sūriken Kōkyūroku 1281, RIMS, Kyoto Univ., 2002, 129140 (in Japanese).

[12] - Vector valued Siegel modular forms of symmetric tensor weight of small degrees, Comment. Math. Univ. St. Pauli 61 (2012), no. 1, to appear.

[13] The Taylor expansion of Jacobi forms of general degree and some applications to explicit structures of higher indices, in Automorphic forms, automorphic representations and related topics, M. Tsuzuki (ed.), Sūriken Kōkyūroku 1715, RIMS, Kyoto Univ., 2010, 169-182.

[14] T. Ibukiyama and R. Kyomura, A generalization of vector valued Jacobi forms, Osaka J. Math. 48 (2011), 783-808. Zbl pre05969050 MR 2837681

[15] J. Igusa, On Siegel modular forms of genus two, Amer. J. Math. 84 (1962), 175-200; (II), ibid. 86 (1964), 392-412. Zbl 0133.33301 MR 0141643(I) MR 0168805(II)

[16] On the graded ring of theta-constants, Amer. J. Math. 86 (1964), 219-246; (II) ibid. 88 (1966), 221-236. Z Zbl 0146.31703(I), Zbl 0146.31704(II) MR 0164967(I) MR 0200482(II)

[17] J. Kramer, Jacobiformen und Thetareihen, Manuscripta Math. 54 (1986), 279-322. Zbl 0588.10024 MR 0819403

[18] D. Mumford, Tata lectures on Theta I, Progr. Math. 28, Birkhäuser Boston, Boston, MA, 1983. Zbl 1112.14002 MR 2352717

[19] T. Satoh, On certain vector valued Siegel modular forms of degree two, Math. Ann. 274 (1986), 335-352. Zbl 0571.10028 MR 0838473

[20] R. Tsushima, The spaces of Siegel cusp forms of degree two and the representation of $\operatorname{Sp}\left(2, F_{p}\right)$, Proc. Japan Acad. Ser. A Math. Sci. 60 (1984), 209-211. Zbl 0573.10017 MR 0758044

[21] _ On the dimension formula for the spaces of Jacobi forms of degree two, in Automorphic forms and L-functions (Kyoto, 1999), Sūriken Kōkyūroku 1103, RIMS, Kyoto Univ., 1999, 96-110. Zbl 0951.11515 MR 1746313

[22] $\_$Dimension formula for the spaces of Jacobi forms of degree two, preprint, 2010, $49 \mathrm{pp}$.

[23] C. Ziegler, Jacobi forms of higher degree, Abh. Math. Sem. Univ. Hamburg 59 (1989), 191-224. Zbl 0707.11035 MR 1049896 